\title{
Mean curvature flow with obstacles: Existence, uniqueness and regularity of solutions*
}

\author{
GWENAEL MERCIER \\ CMAP, École polytechnique, Palaiseau, France \\ E-mail: gwenael.mercier@cmap.polytechnique.fr \\ Matteo Novaga \\ Dipartimento di Matematica, Università di Pisa, Largo Bruno Pontecorvo 5, 56127 Pisa, Italy \\ E-mail:novaga@dm.unipi.it
}

[Received 24 September 2014 and in revised form 12 May 2015]

\begin{abstract}
We show short time existence and uniqueness of $C^{1,1}$ solutions to the mean curvature flow with obstacles, when the obstacles are of class $C^{1,1}$. If the initial interface is a periodic graph we show long time existence of the evolution and convergence to a minimal constrained hypersurface.
\end{abstract}

2010 Mathematics Subject Classification: Primary 53C44; Secondary: 35R45, 49Q05.

Keywords: Mean curvature flow, obstacle problem, short time existence.

\section{Introduction and main results}

Mean curvature flow is a prototypical geometric evolution, arising in many models from Physics, Biology and Material Science, as well as in a variety of mathematical problems. For such a reason, this flow has been widely studied in the past years, starting from the pioneering work of $\mathrm{K}$. Brakke [3] (we refer to [4, 8, 11, 14, 15] for a far from complete list of references).

In some models, one needs to include the presence of hard obstacles, which the evolving surface cannot penetrate (see for instance [12] and references therein). This leads to a double obstacle problem for the mean curvature flow, which reads

$$
v=H \quad \text { on } M_{t} \cap U,
$$

with constraint

$$
M_{t} \subset \bar{U} \quad \text { for all } t,
$$

where $v, H$ denote respectively the normal velocity and $d$ times the mean curvature of the interface $M_{t} \subset \mathbb{R}^{d+1}$, and the closed set $U^{c}$ represents the obstacle. Notice that, due to the presence of obstacles, the evolving interface is in general only of class $C^{1,1}$ in the space variable, differently from the unconstrained case where it is analytic (see [17]). While the regularity of parabolic obstacle problems is relatively well understood (see [23] and references therein), a satisfactory existence and uniqueness theory for solutions is still missing.

* This work is associated with the authors' participation at the "Free Boundary Problems and Related Topics", a scientific programme held at the Isaac Newton Institute of the University of Cambridge, from 6th January 2014 to 4th July 2014, https://www. newton.ac.uk/event/frb. 
In [1] (see also [24]) the authors approximate such an obstacle problem with an implicit variational scheme introduced in $[2,18]$. As a byproduct, they prove global existence of weak (variational) solutions, and short time existence and uniqueness of regular solutions in the twodimensional case. In [20] the first author adapts to this setting the theory of viscosity solutions introduced in $[4,5]$, and constructs globally defined continuous (viscosity) solutions.

Let us now state the main results of this paper.

THEOREM 1 Let $M_{0} \subset U$ be an initial hypersurface, and assume that both $M_{0}$ and $\partial U$ are uniformly of class $C^{1,1}$, with $\operatorname{dist}\left(M_{0}, \partial U\right)>0$. Then there exists $T>0$ and a unique solution $M_{t}$ to (1), (2) on $[0, T)$, such that $M_{t}$ is of class $C^{1,1}$ for all $t \in[0, T)$.

Notice that Theorem 1 extends a result in [1] to dimensions greater than two.

When the hypersurface $M_{t}$ can be written as the graph of a function $u(\cdot, t): \mathbb{R}^{d} \rightarrow \mathbb{R}$, equation (1) reads

$$
u_{t}=\sqrt{1+|\nabla u|^{2}} \operatorname{div}\left(\frac{\nabla u}{\sqrt{1+|\nabla u|^{2}}}\right) .
$$

If the obstacles are also graphs, the constraint (2) can be written as

$$
\psi^{-} \leqslant u \leqslant \psi^{+},
$$

where the functions $\psi^{ \pm}: \mathbb{R}^{d} \rightarrow \mathbb{R}$ denote the obstacles.

Theorem 2 Assume that $\psi^{ \pm} \in C^{1,1}\left(\mathbb{R}^{d}\right)$, and let $u_{0} \in C^{1,1}\left(\mathbb{R}^{d}\right)$ satisfy (4). Then there exists a unique (viscosity) solution $u$ of (3), (4) on $\mathbb{R}^{d} \times[0,+\infty$ ), such that

$$
\begin{aligned}
\|\nabla u(\cdot, t)\|_{L^{\infty}\left(\mathbb{R}^{d}\right)} & \leqslant \max \left(\left\|\nabla u_{0}\right\|_{L^{\infty}\left(\mathbb{R}^{d}\right)},\left\|\nabla \psi^{ \pm}\right\|_{L^{\infty}\left(\mathbb{R}^{d}\right)}\right) \\
\left\|u_{t}(\cdot, t)\right\|_{L^{\infty}\left(\mathbb{R}^{d}\right)} & \leqslant\left\|\sqrt{1+\left|\nabla u_{0}\right|^{2}} \operatorname{div}\left(\frac{\nabla u_{0}}{\sqrt{1+\left|\nabla u_{0}\right|^{2}}}\right)\right\|_{L^{\infty}\left(\mathbb{R}^{d}\right)}
\end{aligned}
$$

for all $t>0$. Moreover $u$ is also of class $C^{1,1}$ uniformly on $[0,+\infty)$.

We observe that Theorem 2 extends previous results by Ecker and Huisken [8] in the unconstrained case (see also [6]).

THEOREM 3 Assume that $u_{0}$ and $\psi^{ \pm}$are $Q$-periodic, with periodicity cell $Q=[0, L]^{d}$, for some $L>0$. Then the solution $u(\cdot, t)$ of (3), (4) is also $Q$-periodic. Moreover there exists a sequence $t_{n} \rightarrow+\infty$ such that $u\left(\cdot, t_{n}\right)$ converges uniformly as $n \rightarrow+\infty$ to a stationary solution to (3), (4).

Our strategy of proof will be to approximate the obstacles with "soft obstacles" modeled by a sequence of uniformly bounded forcing terms. Differently from [1], where the existence of regular solution is derived from variational estimates on the approximating scheme, we obtain estimates on the evolving interface, in the spirit of $[7,9,10]$, which are uniform in the forcing terms.

The plan of the paper is the following: in Section 2 we adapt some well known results on mean curvature flow, such as Huisken's monotonicity formula, to the case of forced mean curvature flow. In Section 3 we prove Theorem 1. In Section 4 we prove Theorem 2. In Section 5 we prove Theorem 3. Eventually, in the Appendix at the end of the paper we adapt the concept of viscosity solution in order to treat the case of mean curvature flow of graphs in the presence of obstacles. 
Acknowledgements, We wish to thank to Antonin Chambolle for interesting discussions and useful comments. The first author was partially supported by the ANR-12-BS01-0014-01 Project Geometrya. The second author was partially supported by the Italian CNR-GNAMPA and by the University of Pisa via grant PRA-2015-0017.

\section{Mean curvature flow with a forcing term}

\subsection{Evolution of geometric quantities}

Let $M$ be a complete orientable $d$-dimensional Riemannian manifold without boundary, let $F(\cdot, t)$ : $M \rightarrow \mathbb{R}^{d+1}$ be a smooth family of immersions, and denote by $M_{t}$ the image $F(M, t)$. Since $M_{t}$ is orientable, we can write $M_{t}=\partial E(t)$ where $E(t)$ is a family of open subsets of $\mathbb{R}^{d+1}$ depending smoothly on $t$. We say that $M_{t}$ evolves by mean curvature with forcing term $k$ if

$$
\frac{d}{d t} F(p, t)=-(H(p, t)+k(F(p, t))) v(p, t),
$$

where $k: \mathbb{R}^{d+1} \rightarrow \mathbb{R}$ is a smooth forcing term, $v$ is the unit normal to $M_{t}$ pointing outside $E(t)$, and $H$ is ( $d$ times) the mean curvature of $M_{t}$, with the convention that $H$ is positive whenever $E(t)$ is convex.

We shall compute the evolution of some relevant geometric quantities under the law (5). We denote by $\nabla^{S}, \Delta^{S}$ respectively the covariant derivative and the Laplace-Beltrami operator on $M$. As in [15], the metric on $M_{t}$ is denoted by $g_{i j}(t)$, it inverse is $g^{i j}(t)$, the scalar product (or any tensors contraction using the metric) on $M_{t}$ is denoted by $\langle\cdot, \cdot\rangle$ whereas the ambient scalar product is $(\cdot, \cdot)$, the volume element is $\mu_{t}$, and the second fundamental form is $A$. In particular we have $A\left(\partial_{i}, \partial_{j}\right)=h_{i j}$, where we set for simplicity $\partial_{i}=\frac{\partial}{\partial x_{i}}$, and $H=g^{i j} h_{i j}$, using the Einstein notation (we implicitly sum over every index which appears twice). We also denote by $\lambda_{1}, \ldots, \lambda_{d}$ the eigenvalues of $A$.

Notice that, in terms of the parametrization $F$, we have

$$
g_{i j}=\left(\partial_{i} F, \partial_{j} F\right), \quad h_{i j}=-\left(\partial_{i j}^{2} F, v\right) \quad \text { for all } i, j \in\{1, \ldots, d\} .
$$

PROPOSITION 1 The following equalities hold:

$$
\begin{aligned}
\frac{d}{d t} g_{i j} & =-2(H+k) h_{i j} \\
\frac{d}{d t} v & =\nabla^{S}(H+k) \\
\frac{d}{d t} \mu_{t} & =-H(H+k) \mu_{t} \\
\frac{d}{d t} h_{i j} & =\Delta^{S} h_{i j}+\nabla_{i}^{S} \nabla_{j}^{S} k-2 H h_{i l} g^{l m} h_{m j}-k g^{m l} h_{i m} h_{j l}+|A|^{2} h_{i j} \\
\frac{d}{d t} H & =\Delta^{S}(H+k)+(H+k)|A|^{2} \\
\frac{d}{d t}|A|^{2} & =\Delta^{S}|A|^{2}+2 k g^{i j} g^{s l} g^{m n} h_{i s} h_{l m} h_{n j}+2|A|^{4}-2\left|\nabla^{S} A\right|^{2}+2\left\langle A,\left(\nabla^{S}\right)^{2} k\right\rangle .
\end{aligned}
$$


Proof. The proof follows by direct computations as in [10, 15]. Recalling (6), we get

$$
\begin{aligned}
\frac{d}{d t} g_{i j} & =\frac{d}{d t}\left(\partial_{i} F, \partial_{j} F\right)=-(H+k)\left(\left(\partial_{i} v, \partial_{j} F\right)+\left(\partial_{i} F, \partial_{j} v\right)\right)=-2(H+k) h_{i j} \\
\frac{d}{d t} v & =\left(\frac{d}{d t} v, \partial_{i} F\right) g^{i j} \partial_{j} F=-\left(v, \frac{d}{d t} \partial_{i} F\right) g^{i j} \partial_{j} F \\
& =\left(v, \partial_{i}((H+k) v)\right) g^{i j} \partial_{j} F=\partial_{i}(H+k) g^{i j} \partial_{j} F=\nabla^{S}(H+k) .
\end{aligned}
$$

The evolution of the measure on $M_{t}$

$$
\mu_{t}=\sqrt{\operatorname{det}[g]}
$$

is given by

$$
\begin{aligned}
\frac{d}{d t} \sqrt{\operatorname{det}[g]} & =\frac{\frac{d}{d t} \operatorname{det}[g]}{2 \sqrt{\operatorname{det}[g]}}=\frac{\operatorname{det}[g] \cdot \operatorname{Tr}\left(g^{i j} \frac{d}{d t} g_{i j}\right)}{2 \sqrt{\operatorname{det}[g]}} \\
& =-\sqrt{\operatorname{det}[g]} \cdot(H+k) g^{i j} h_{j i}=-\mu_{t} H(H+k) .
\end{aligned}
$$

In order to prove (10) we compute (as usual, we denote the Christoffel symbols by $\Gamma_{i j}^{k}$ )

$$
\begin{aligned}
\frac{d}{d t} h_{i j}= & -\frac{d}{d t}\left(v, \partial_{i j}^{2} F\right) \\
= & -\left(\nabla^{S}(H+k), \partial_{i j}^{2} F\right)+\left(\partial_{i j}^{2}(H+k) v, v\right) \\
= & -\left(g^{k l} \partial_{k}(H+k) \partial_{l} F, \Gamma_{i j}^{k} \partial_{k} F-h_{i j} v\right) \\
& \quad+\partial_{i j}^{2}(H+k)+(H+k)\left(\partial_{j}\left(h_{i m} g^{m l} \partial_{l} F\right), v\right) \\
= & \partial_{i j}^{2}(H+k)-\Gamma_{i j}^{k} \partial_{k}(H+k)+(H+k) h_{i m} g^{m l}\left(\Gamma_{l j}^{k} \partial_{k} F-h_{l j} v, v\right) \\
= & \nabla_{i}^{S} \nabla_{j}^{S}(H+k)-(H+k) h_{i l} g^{l m} h_{m j} .
\end{aligned}
$$

Using Codazzi's equations, one can show that

$$
\Delta^{S} h_{i j}=\nabla_{i}^{S} \nabla_{j}^{S} H+H h_{i l} g^{l m} h_{m j}-|A|^{2} h_{i j},
$$

so that (10) follows from (14) and (13). From (10) we deduce

$$
\begin{aligned}
\frac{d}{d t} H & =\frac{d}{d t} g^{i j} h_{i j} \\
& =2(H+k) g^{i s} h_{s l} g^{l j} h_{i j}+g^{i j}\left(\nabla_{i}^{S} \nabla_{j}^{S}(H+k)-(H+k) h_{i l} g^{l m} h_{m j}\right) \\
& =\Delta^{S}(H+k)+(H+k)|A|^{2},
\end{aligned}
$$


which gives (11). In addition, we get

$$
\begin{aligned}
\frac{d}{d t}|A|^{2}= & \frac{d}{d t}\left(g^{i k} g^{j l} h_{i j} h_{k l}\right) \\
= & 2 \frac{d}{d t} g^{j l} h_{i j} h_{k l}+2 g^{i k} g^{j l} \frac{d}{d t} h_{i j} h_{k l} \\
= & 2\left(2(H+k) g^{j s} h_{s t} g^{t l}\right) g^{j l} h_{i j} h_{k l} \\
& \quad+2 g^{i k} g^{j l}\left(\Delta^{S} h_{i j}+\nabla_{i}^{S} \nabla_{j}^{S} k-2 H h_{i l} g^{l m} h_{m j}-k g^{m l} h_{i m} h_{j l}+|A|^{2} h_{i j}\right) h_{k l} \\
= & 2 k g^{j s} h_{s t} g^{t l} g^{j l} h_{i j} h_{k l}+2 g^{i k} g^{j l} \Delta^{S} h_{i j} h_{k l}+2|A|^{4}+2\left\langle A,\left(\nabla^{S}\right)^{2} k\right\rangle .
\end{aligned}
$$

On the other hand, one has

$$
\Delta^{S}|A|^{2}=2\left\langle\Delta^{S} A, A\right\rangle+2\left|\nabla^{S} A\right|^{2}=2 g^{p q} g^{m n} h_{p m} \Delta^{S} h_{q n}+2\left|\nabla^{S} A\right|^{2} .
$$

so that (12) follows from (16) and (15).

\subsection{The monotonicity formula}

We extend Huisken's monotonicity formula [16] to the forced mean curvature flow (5) (see also [7, Section 2.2]).

Given a vector field $\omega: M_{t} \rightarrow \mathbb{R}^{d+1}$, we let

$$
\omega^{\perp}=(\omega, v) v, \quad \omega^{T}=\omega-\omega^{\perp} .
$$

Letting $X_{0} \in \mathbb{R}^{d+1}$ and $t_{0} \in \mathbb{R}$, for $(x, t) \in \mathbb{R}^{d+1} \times\left[t_{0},+\infty\right)$ we define the kernel

$$
\rho(x, t)=\frac{1}{\left(4 \pi\left(t_{0}-t\right)\right)^{d / 2}} \exp \left(\frac{-\left|x-x_{0}\right|^{2}}{4\left(t_{0}-t\right)}\right) .
$$

A direct computation gives

$$
\frac{d \rho}{d t}=-\Delta^{S} \rho+\rho\left(\frac{\left(x_{0}-x,(H+k) v\right)}{t_{0}-t}-\frac{\left|\left(x_{0}-x\right)^{\perp}\right|^{2}}{4\left(t_{0}-t\right)^{2}}\right) .
$$

Proposition 2 (Monotonicity formula)

$$
\frac{d}{d t} \int_{M_{t}} \rho=-\int_{M_{t}} \rho\left(\left|H+\frac{k}{2}+\frac{\left(x-x_{0}, v\right)}{2\left(t_{0}-t\right)}\right|^{2}-\frac{k^{2}}{4}\right) .
$$


Proof. Recalling (9), we compute

$$
\begin{aligned}
\frac{d}{d t} \int_{M_{t}} \rho & =\int_{M_{t}} \frac{d}{d t} \rho-H(H+k) \rho \\
& =\int_{M_{t}} \rho\left(-\frac{\left|x-x_{0}\right|^{2}}{4\left(t_{0}-t\right)^{2}}+\frac{d}{2\left(t_{0}-t\right)}-\frac{\left(x-x_{0}, v\right)}{2\left(t_{0}-t\right)}(H+k)-H(H+k)\right) \\
& =-\int_{M_{t}} \rho\left(\left|H v+\frac{x-x_{0}}{2\left(t_{0}-t\right)}+\frac{k v}{2}\right|^{2}-\frac{k^{2}}{4}\right)+\int_{M_{t}} \frac{d}{2\left(t_{0}-t\right)} \rho \\
& \quad+\int_{M_{t}} \frac{\rho\left(x-x_{0}, v\right) H}{2\left(t_{0}-t\right)}
\end{aligned}
$$

We use the first variation formula: for all vector field $Y$ on $M_{t}$, we have

$$
\int_{M_{t}} \operatorname{div}_{M_{t}} Y=\int_{M_{t}}\langle H v, Y\rangle \text {. }
$$

As a result, with $Y=\frac{\rho\left(x-x_{0}\right)}{2\left(t-t_{0}\right)}$, we get

$$
\begin{aligned}
\frac{d}{d t} \int_{M_{t}} \rho & =-\int_{M_{t}} \rho\left(\left|H v+\frac{x-x_{0}}{2\left(t_{0}-t\right)}+\frac{k v}{2}\right|^{2}-\frac{k^{2}}{4}-\frac{\left|\left(x-x_{0}\right)^{T}\right|^{2}}{4\left(t_{0}-t\right)^{2}}\right) \\
& =-\int_{M_{t}} \rho\left(\left|H+\frac{\left(x-x_{0}, v\right)}{2\left(t_{0}-t\right)}+\frac{k}{2}\right|^{2}-\frac{k^{2}}{4}\right) .
\end{aligned}
$$

In a similar way (see [8]) one can prove that for all functions $f(X, t)$ defined on $M_{t}$, one has

$$
\partial_{t} \int_{M_{t}} \rho f=\int_{M_{t}}\left(\frac{d f}{d t}-\Delta^{S} f\right) \rho-\int_{M_{t}} f \rho\left(\left|H+\frac{\left(x-x_{0}, v\right)}{2\left(t_{0}-t\right)}+\frac{k}{2}\right|^{2}-\frac{k^{2}}{4}\right) .
$$

Indeed, using (17)

$$
\begin{aligned}
\frac{d}{d t} & \int_{M_{t}} \rho f=\int_{M_{t}} f \frac{d \rho}{d t}+\frac{d f}{d t} \rho-H(H+k) f \rho \\
& =\int_{M_{t}} f\left(\frac{d \rho}{d t}-H(H+k) \rho\right)+\frac{d f}{d t} \rho \\
& =\int_{M_{t}} f\left(-\Delta^{S} \rho+\rho\left(\frac{\left(X_{0}-X,(H+k) v\right)}{t_{0}-t}-\frac{1}{4} \frac{\left|\left(X_{0}-X\right)^{\perp}\right|^{2}}{\left(t_{0}-t\right)^{2}}\right)-H(H+k) \rho\right)+\frac{d f}{d t} \rho \\
& =\int_{M_{t}}-\Delta^{S} f \rho+\left(\rho\left(\frac{\left(X_{0}-X,(H+k) v\right)}{t_{0}-t}-\frac{1}{4} \frac{\left|\left(X_{0}-X\right)^{\perp}\right|^{2}}{\left(t_{0}-t\right)^{2}}\right)-H(H+k) \rho\right)+\frac{d f}{d t} \rho \\
& =\int_{M_{t}} \rho\left(\frac{d}{d t} f-\Delta^{S} f\right)-\int f \rho\left(\left|H+\frac{\left(x-x_{0}, v\right)}{2\left(t_{0}-t\right)}+\frac{k}{2}\right|^{2}-\frac{k^{2}}{4}\right) .
\end{aligned}
$$


LEMMA 1 Let $f$ be defined on $M_{t}$ and satisfy

$$
\frac{d}{d t} f-\Delta^{S} f \leqslant a \cdot \nabla^{S} f \quad \text { on } M_{t}
$$

for some vector field $a$ bounded on $\left[0, t_{1}\right]$. Then,

$$
\sup _{M_{t}, t \in\left[0, t_{1}\right]} f \leqslant \sup _{M_{0}} f \text {. }
$$

Proof. Denote by $a_{0}$ the bound on $a, k:=\sup _{M_{0}} f$ and define $f_{l}=\max (f-l, 0)$. Assumption (19) implies

$$
\left(\frac{d}{d t}-\Delta^{S}\right) f_{l}^{2} \leqslant 2 f_{l} a \cdot \nabla^{S} f_{l}-2\left|\nabla^{S} f_{l}\right|^{2}
$$

which, thanks to Young's inequality, gives

$$
\left(\frac{d}{d t}-\Delta^{S}\right) f_{l}^{2} \leqslant \frac{1}{2} a_{0}^{2} f_{l}^{2}
$$

Applying (18) to $f_{l}^{2}$, we get

$$
\frac{d}{d t} \int f_{l}^{2} \rho \leqslant \frac{1}{2}\left(a_{0}^{2}+\|k\|_{\infty}^{2}\right) \int f_{l}^{2} \rho .
$$

Letting $l=\sup _{M_{0}} f$, so that $f_{l} \equiv 0$ on $M_{0}$, from (20) and the Gronwall's Lemma we obtain that $f_{l} \equiv 0$ on $M_{t}$ for all $t \in\left(0, t_{1}\right]$, which gives thesis.

\section{Proof of Theorem 1}

We now prove short time existence for the mean curvature flow with obstacles (1), (2). Let $M_{0}=$ $\partial E(0) \subset U$, where we assume that $U, E(0)$ are open sets with boundary uniformly of class $C^{1,1}$. In particular, $M_{0}$ satisfies a uniform exterior and interior ball condition, that is, there is $R>0$ such that, for every $x \in M_{0}$, one can find two open balls $B^{+}$and $B^{-}$of radius $R$ which are tangent to $M_{0}$ at $x$ and such that $B^{+} \subset E(0)^{c}$ and $B^{-} \subset E(0)$. Let also $\Omega^{-}:=E(0) \backslash \bar{U}$, and $\Omega^{+}:=E(0) \cup U$. Notice that $\Omega^{ \pm}$are open sets with $C^{1,1}$ boundaries, with $\operatorname{dist}\left(\Omega^{-}, \partial \Omega^{+}\right)>0$. Note that the condition $M_{t} \subset \bar{U}$ can be rewritten as

$$
\Omega^{-} \subset E(t) \subset \Omega^{+} .
$$

Let also

$$
k:=2 N\left(1-\chi_{\Omega^{+}}-\chi_{\Omega^{-}}\right)
$$

where $N$ is bigger than ( $d$ times) the mean curvature of $\partial U$.

We want to show that equation (5), with $k$ as above, has a solution in an interval $[0, T)$. To this purpose, letting $\rho_{\varepsilon}$ be a standard mollifier supported in the ball of radius $\varepsilon$ centered at 0 , we introduce a smooth regularization $k_{\varepsilon}=k * \rho_{\varepsilon}$ of $k$. Notice that $\left\|k_{\varepsilon}\right\|_{\infty}=2 N, k_{\varepsilon}(x)=-2 N$ (resp. $\left.k_{\varepsilon}(x)=2 N\right)$ at every $x \in \Omega^{-}$(resp. $x \notin \Omega^{+}$) such that $\operatorname{dist}(x, \partial U) \geqslant \varepsilon$, and $k_{\varepsilon}(x)=0$ at every $x \in U$ such that $\operatorname{dist}(x, \partial U) \geqslant \varepsilon$. 
Using standard arguments (see for instance [10, Theorem 4.1] and [9, Prop. 4.1]) one can show existence of a smooth solution $M_{t}^{\varepsilon}$ of (5), with $k$ replaced by $k_{\varepsilon}$, on a maximal time interval $\left[0, T_{\varepsilon}\right.$ ).

Let now

$$
\Omega_{\varepsilon}^{-}:=\left\{x \in \Omega^{-}: \operatorname{dist}\left(x, \partial \Omega^{-}\right)>\varepsilon\right\}
$$

and

$$
\Omega_{\varepsilon}^{+}:=\left\{x \in \mathbb{R}^{d+1}: \operatorname{dist}\left(x, \Omega^{+}\right)<\varepsilon\right\} .
$$

The following result follows directly from the definition of $k_{\varepsilon}$.

PROPOSITION 3 The hypersurfaces $\partial \Omega_{\varepsilon}^{-}$and $\partial\left(\Omega_{\varepsilon}^{+}\right)^{c}$ are respectively a super and a subsolution of (5), with $k$ replaced with $k_{\varepsilon}$. In particular, by the parabolic comparison principle $M_{t}^{\varepsilon}$ cannot intersect $\partial \Omega_{\varepsilon}^{ \pm}$.

We will show that we can find a time $T>0$ such that for every $\varepsilon$, there exists a smooth solution of (5) (with $k$ replaced with $k_{\varepsilon}$ ) on $[0, T$ ).

The following result will be useful in the sequel. We omit the proof which is a simple ODE argument.

LEMMA 2 Let $M_{0}=\partial B_{R}\left(x_{0}\right)$ be a ball of radius $R \leqslant 1$ centered at $x_{0}$. Then, the evolution $M_{t}$ by (5), with constant forcing term $k=2 N$, is given by $M_{t}=B_{R(t)}\left(x_{0}\right)$ with $R(t) \geqslant$ $\sqrt{R^{2}-(4 N+2 d) t}$. In particular, the solution exists at least on $\left[0, \frac{R^{2}}{4 N+2 d}\right)$.

PRoposition 4 There exists $r>0$, a collection of balls $B_{i}=B_{r}\left(x_{i}\right)$ of radius $r$, and a positive time $T_{0}$ such that $M_{t}^{\varepsilon} \subset \bigcup_{i} B_{i}$ for every $t \in\left[0, \min \left(T_{0}, T_{\varepsilon}\right)\right)$. In addition, we can choose the balls $B_{i}$ in such a way that, for every $i$, there exists $\omega_{i} \in \mathbb{R}^{d+1}$ such that the sets $\partial \Omega^{ \pm} \cap B_{4 r}\left(x_{i}\right)$, if nonempty, are graphs of some functions $\psi_{i}^{ \pm}: \mathbb{R}^{d} \rightarrow \mathbb{R}$ over $\omega_{i}^{\perp}$.

In particular, one has

$$
\left(\nabla k_{\varepsilon}, \omega_{i}\right) \geqslant\left|\nabla k_{\varepsilon}\right| / 2 \text { on } B_{2 r}\left(x_{i}\right) .
$$

Most of this notation is summarized in Figure 1.

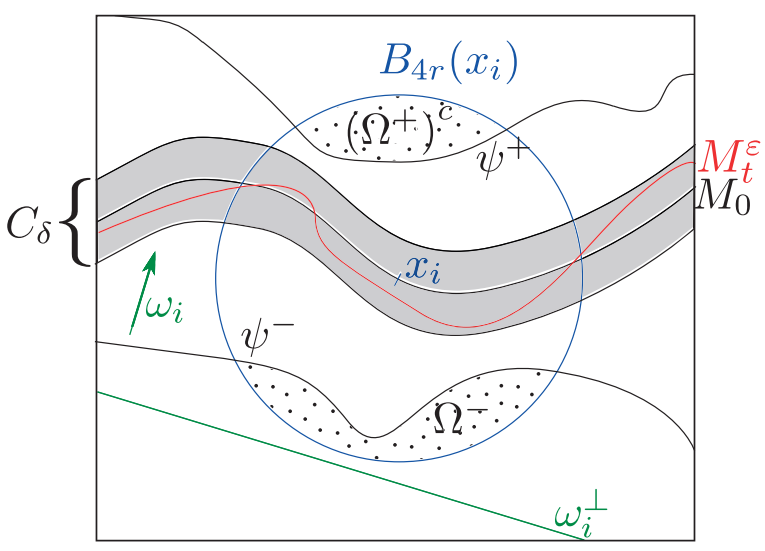

FIG. 1. Notation in Proposition 4 
Proof. By assumption, for every $\mathbf{x} \in M_{0}$ there exist interior and exterior balls $B_{x}^{ \pm}$of fixed radius $R \leqslant 1$. Let $B_{x}^{ \pm}(t)$ be the evolution of $B_{x}^{ \pm}$by (5) with forcing term $k=2 N$. By comparison, for every $t \in\left[0, T_{\varepsilon}\right), B_{x}^{+}(t) \subset \Omega(t)^{c}$ and $B_{x}^{-}(t) \subset \bar{\Omega}(t)$. Recalling Lemma 2, there exists $\delta>0$ and $T_{0}>0$, independent of $\varepsilon$, such that $M_{t} \subset\left\{d_{M_{0}} \leqslant \delta\right\}=: C_{\delta}$, for all $t \in\left[0, \min \left(T_{\varepsilon}, T_{0}\right)\right)$.

We eventually reduce $\delta, T_{0}$ such that $C_{\delta}$ can be covered with a collection of balls $B_{i}=B_{r}\left(x_{i}\right)$, centered at $x_{i} \in M_{0}$ and with a radius $r$ such that, for every $i$, there exists a unit vector $\omega_{i} \in \mathbb{R}^{d+1}$ satisfying

$$
\left(\omega_{i}, v^{+}(x)\right) \geqslant \frac{1}{2} \quad \text { and } \quad\left(\omega_{i}, v^{-}(y)\right) \geqslant \frac{1}{2}
$$

for every $x \in \partial \Omega^{+} \cap B_{4 r}\left(x_{i}\right)$ and $y \in \partial \Omega^{-} \cap B_{4 r}\left(x_{i}\right)$, where $v^{ \pm}$is the outer normal to $\Omega^{ \pm}$.

As a result, $\partial \Omega^{ \pm} \cap B_{4 r}\left(x_{i}\right)$ are graphs of some functions $\psi_{i}^{ \pm}: \mathbb{R}^{d} \rightarrow \mathbb{R}$ over $\omega_{i}^{\perp}$ (see Figure 1).

Notice also that $k$ is a $B V$ function and $D k$ is a Radon measure concentrated on $\partial U$ such that

$$
\left(D k, \omega_{i}\right) \geqslant \frac{|D k|}{2} \text { on } B_{4 r}\left(x_{i}\right) .
$$

Then, for every $x \in B_{2 r}\left(x_{i}\right)$ and $\varepsilon$ sufficiently small (such that $\rho_{\varepsilon}(x)=0$ as soon as $|x| \geqslant 2 r$ ), we have

$$
\begin{aligned}
\left(\nabla k_{\varepsilon}, \omega_{i}\right) & =\left(\nabla \int_{\mathbb{R}^{d+1}} k(x-y) \rho_{\varepsilon}(y) d y, \omega_{i}\right) \\
& =\int_{\mathbb{R}^{d+1}}\left(D k(x-y), \omega_{i}\right) \rho_{\varepsilon}(y) d y \\
& \geqslant \int_{\mathbb{R}^{d+1}} \frac{|D k|(x-y)}{2} \rho_{\varepsilon}(y) d y \\
& \geqslant \frac{|D k| * \rho_{\varepsilon}}{2} \geqslant \frac{\left|\nabla k_{\varepsilon}\right|}{2} .
\end{aligned}
$$

In what follows, we will control the geometric quantities of $M_{t}^{\varepsilon}$ inside each ball $B_{i}$. As in [9], we introduce a localization function $\phi_{i}$ as follows: let $\eta_{i}(x, t)=\left|x-x_{i}\right|^{2}+(2 d+\Lambda) t(\Lambda$ be a positive constant that will be fixed later) and, for $R=2 r, \phi_{i}(x, t)=\left(R^{2}-\eta_{i}(x, t)\right)^{+}$. We denote by $\phi_{i}$ the quantity $\phi_{i}(\mathbf{x}, t)$, where $\mathbf{x}=\mathbf{x}(p, t)$ will be a generic point in $M_{t}$. Notice that there exists $T_{1}=\frac{r^{2}}{2 d+\Lambda}$ such that for all $t \in\left[0, \min \left(T_{1}, T_{\varepsilon}\right)\right)$,

$$
M_{t}^{\varepsilon} \subset \bigcup_{i}\left\{\phi_{i}>r^{2}\right\} .
$$

As a result, we have the following

Lemma 3 Let $f$ be a smooth function defined on $M_{t}^{\varepsilon}$. Assume that there is a $C>0$ such that

$$
\phi_{i} f \leqslant C \text { on } M_{t}^{\varepsilon} \quad \forall t \leqslant \min \left(T_{\varepsilon}, T_{1}\right) \text { and } \forall i \in \mathbb{N} .
$$

Then,

$$
f \leqslant \alpha C \text { on } M_{t}^{\varepsilon} \quad \forall t \leqslant \min \left(T_{\varepsilon}, T_{1}\right),
$$

where $\alpha$ depends only on the $C^{1,1}$ norm of $M_{0}$. 
LEMMA 4 Let $v:=(v, \omega)^{-1}$. The quantity $v^{2} \phi^{2}$ satisfies

$$
\left(\frac{d}{d t}-\Delta^{S}\right)\left(\frac{v^{2} \phi^{2}}{2}\right) \leqslant \frac{1}{2}\left(\nabla^{S}\left(v^{2} \phi^{2}\right), \frac{\nabla^{S} \phi^{2}}{\phi^{2}}\right)-\phi^{2} v^{3}\left(\nabla^{S} k_{\varepsilon}, \omega\right)+v^{2} \phi\left(2 k_{\varepsilon}(\mathbf{x}, v)-\Lambda\right) .
$$

Proof. In this proof and the proofs further, we use normal coordinates: we assume that $g_{i j}=\delta_{i j}$ (Kronecker symbol) and that the Christoffel symbols $\Gamma_{i j}^{k}$ vanish at the computation point.

We expand the derivatives

$$
\left(\frac{d}{d t}-\Delta^{S}\right)\left(\frac{v^{2} \phi^{2}}{2}\right)=v^{2}\left(\frac{d}{d t}-\Delta^{S}\right) \frac{\phi^{2}}{2}+\phi^{2}\left(\frac{d}{d t}-\Delta^{S}\right) \frac{v^{2}}{2}-2\left\langle\nabla^{S} \frac{\phi^{2}}{2}, \nabla^{S} \frac{v^{2}}{2}\right\rangle
$$

First term. We start computing

$$
\left(\frac{d}{d t}-\Delta^{S}\right)|\mathbf{x}|^{2}=-2 k_{\varepsilon}(\mathbf{x}, v)-2 d
$$

Then,

$$
\left(\frac{d}{d t}-\Delta^{S}\right) \phi^{2}=2 \phi\left(2 k_{\varepsilon}\left(\mathbf{x}-x_{i}, v\right)-\Lambda\right)-\left.\left.2\left|\nabla^{S}\right| \mathbf{x}\right|^{2}\right|^{2}
$$

Second term. We are interested in

$$
\begin{aligned}
\frac{1}{2} \frac{d}{d t}(\omega, v)^{2} & =(\omega, v)\left(\frac{d}{d t} v, \omega\right) \\
& =(\omega, v)\left(\nabla^{S}\left(H+k_{\varepsilon}\right), \omega\right) .
\end{aligned}
$$

So,

$$
\frac{1}{2} \frac{d}{d t}(\omega, v)^{-2}=-(\omega, v)^{-3}\left(\nabla^{S}\left(H+k_{\varepsilon}\right), \omega\right)
$$

On the other hand,

$$
\frac{1}{2} \Delta^{S}\left((\omega, v)^{-2}\right)=(\omega, v)^{-1} \Delta^{S}(\omega, v)^{-1}-\left\langle\nabla^{S}(\omega, v)^{-1}, \nabla^{S}(\omega, v)^{-1}\right\rangle
$$

Let us note that

$$
\partial_{i j} v=\partial_{i}\left(h_{j l} g^{l m} \partial_{m} F\right)=\partial_{i}\left(h_{j l}\right) \delta_{l m} \partial_{m} F-h_{j l} \delta_{l m}\left(-h_{i m} v\right)=\partial_{i}\left(h_{j l}\right) \partial_{l} F-\lambda_{i}^{2} \delta_{i j} v .
$$

We then get

$$
\begin{aligned}
\Delta^{S}(\omega, v)^{-1} & =\partial_{i i}(\omega, v)^{-1}=\partial_{i}\left(-\left(\omega, \partial_{i} v\right)(\omega, v)^{-2}\right) \\
& =-\left(\omega, \partial_{i i} v\right)(\omega, v)^{-2}+2\left(\omega, \partial_{i} v\right)^{2}(\omega, v)^{-3} \\
& =-(\omega, v)^{-2}\left(\partial_{i} h_{i l} \partial_{l} F-\lambda_{i}^{2} v, \omega\right)+2(\omega, v)^{-3}\left(\omega, \lambda_{i} \partial_{i} F\right)^{2} . \\
& =-(\omega, v)^{-2}\left(\partial_{l} h_{i i} \partial_{l} F, \omega\right)+|A|^{2}(v, \omega)^{-1}+2(\omega, v)^{-3}\left(\omega, \lambda_{i} \partial_{i} F\right)^{2} .
\end{aligned}
$$


We also have

$$
\begin{aligned}
\left\langle\nabla^{S}(\omega, v)^{-1}, \nabla^{S}(\omega, v)^{-1}\right\rangle & =(\omega, v)^{-4}\left(\omega, \partial_{k} v\right)\left(\omega, \partial_{k} v\right) \\
& =(\omega, v)^{-4}\left(\omega, h_{k u} g^{u v} \partial_{v} F\right)^{2}=(\omega, v)^{-4}\left(\omega, \lambda_{k} \partial_{k} F\right)^{2}
\end{aligned}
$$

which leads to

$$
\begin{aligned}
\left(\frac{d}{d t}-\Delta^{S}\right) \frac{v^{2}}{2}=-v^{3}\left(\nabla^{S}\left(H+k_{\varepsilon}\right), \omega\right) & +v^{3} \partial_{m}\left(h_{i i}\right)\left(\omega, \partial_{m} F\right) \\
- & |A|^{2} v^{2}-2 v^{4} \lambda_{k}^{2}\left(\omega, \partial_{k} F\right)^{2}-v^{4}\left(\omega, \lambda_{k} \partial_{k} F\right)^{2} .
\end{aligned}
$$

Third term. We notice, as in [9] that $\left|\nabla^{S} \phi^{2}\right|^{2}=4 \phi^{2}\left|\nabla^{S}\left(|\mathbf{x}|^{2}\right)\right|^{2}$ and

$$
-\left(\nabla^{S}\left(v^{2}\right), \nabla^{S} \phi^{2}\right)=-3\left(v \nabla^{S}(v), \nabla^{S} \phi^{2}\right)+\frac{1}{2}\left(\left(\nabla^{S}\left(v^{2} \phi^{2}\right), \frac{\nabla^{S} \phi^{2}}{\phi^{2}}\right)-v^{2} \frac{\left|\nabla^{S} \phi^{2}\right|^{2}}{\phi^{2}}\right) .
$$

Then, Young's inequality gives

$$
\begin{aligned}
2\left|v\left(\nabla^{S} v, \nabla^{S} \phi^{2}\right)\right| & \leqslant 2 \phi^{2}\left|\nabla^{S} v^{2}\right|^{2}+\frac{1}{2 \phi^{2}}\left|\nabla^{S} \phi^{2}\right|^{2} \\
& \leqslant 2 \phi^{2}\left|\nabla^{S} v^{2}\right|^{2}+\left.\left.2 v^{2}\left|\nabla^{S}\right| \mathbf{x}\right|^{2}\right|^{2} .
\end{aligned}
$$

Hence,

$$
\left(\nabla^{S}\left(v^{2}\right), \nabla^{S} \phi^{2}\right) \leqslant-3 \phi^{2}\left|\nabla^{S} v^{2}\right|^{2}-\left.\left.3 v^{2}\left|\nabla^{S}\right| \mathbf{x}\right|^{2}\right|^{2}+\frac{1}{2}\left(\left(\nabla^{S}\left(v^{2} \phi^{2}\right), \frac{\nabla^{S} \phi^{2}}{\phi^{2}}\right)-v^{2} \frac{\left|\nabla^{S} \phi^{2}\right|^{2}}{\phi^{2}}\right) .
$$

Summing the three terms, we get

$$
\left(\frac{d}{d t}-\Delta^{S}\right)\left(\frac{v^{2} \phi^{2}}{2}\right) \leqslant \frac{1}{2}\left(\nabla^{S}\left(v^{2} \phi^{2}\right), \frac{\nabla^{S} \phi^{2}}{\phi^{2}}\right)-\phi^{2} v^{3}\left(\nabla^{S} k_{\varepsilon}, \omega\right)+v^{2} \phi\left(2 k_{\varepsilon}(\mathbf{x}, v)-\Lambda\right) .
$$

For $\gamma>0$, we let

$$
\psi\left(v^{2}\right):=\frac{\gamma v^{2}}{1-\gamma v^{2}}
$$

LEMMA 5 For $\varepsilon \leqslant r$, we have

$$
\begin{aligned}
\left(\frac{d}{d t}-\Delta^{S}\right) \frac{\phi^{2}|A|^{2} \psi\left(v^{2}\right)}{2} & \leqslant \phi^{2} \psi\left(v^{2}\right)\left(-\gamma|A|^{4}-2 k_{\varepsilon} \sum_{i} \lambda_{i}^{3}-2\left\langle A,\left(\nabla^{S}\right)^{2} k_{\varepsilon}\right\rangle\right) \\
& -\phi^{2}|A|^{2} v^{3} \psi^{\prime}\left(v^{2}\right)\left(\nabla^{S} k_{\varepsilon}, \omega\right)-\phi^{2}|A|^{2} \sum_{i}\left(\lambda_{i} \omega^{i}\right)^{2} \frac{2 v^{4}+\gamma v^{6}}{\left(1-\gamma v^{2}\right)^{3}}
\end{aligned}
$$


Proof. We denote $V=\frac{\phi^{2}|A|^{2} \psi\left(v^{2}\right)}{2}$ and compute

$$
\begin{aligned}
\left(\frac{d}{d t}-\Delta^{S}\right) \frac{\phi^{2}|A|^{2} \psi\left(v^{2}\right)}{2} & =|A|^{2} \psi\left(v^{2}\right)\left(\frac{d}{d t}-\Delta^{S}\right) \frac{1}{2} \phi^{2}+\phi^{2} \psi\left(v^{2}\right)\left(\frac{d}{d t}-\Delta^{S}\right) \frac{1}{2}|A|^{2} \\
+\phi^{2}|A|^{2} & \left(\frac{d}{d t}-\Delta^{S}\right) \frac{1}{2} \psi\left(v^{2}\right)-2\left\langle 1 / 2 \nabla^{S}|A|^{2}, 1 / 2 \nabla^{S} \phi^{2}\right\rangle \\
& -2\left\langle 1 / 2 \nabla^{S}|A|^{2}, 1 / 2 \nabla^{S} \psi\left(v^{2}\right)\right\rangle-2\left\langle 1 / 2 \nabla^{S} \phi^{2}, 1 / 2 \nabla^{S} \psi\left(v^{2}\right)\right\rangle .
\end{aligned}
$$

The two first terms have already been computed. Let us consider the third one.

$$
\begin{aligned}
\frac{1}{2} & \frac{d}{d t} \psi\left(v^{2}\right)=v \frac{d v}{d t} \psi^{\prime}\left(v^{2}\right)=-v^{3} \psi^{\prime}\left(v^{2}\right)\left(\nabla^{S}\left(H+k_{\varepsilon}\right), \omega\right) \\
\frac{1}{2} \Delta^{S} \psi\left(v^{2}\right) & =\frac{1}{2} \partial_{i i} \psi\left(v^{2}\right)=\partial_{i}\left(v \partial_{i} v \psi^{\prime}\left(v^{2}\right)\right) \\
& =v \Delta^{S} v \psi^{\prime}\left(v^{2}\right)+2 v^{2}\left|\nabla^{S} v\right|^{2} \psi^{\prime \prime}\left(v^{2}\right)+\left|\nabla^{S} v\right|^{2} \psi^{\prime}\left(v^{2}\right) \\
& =\left(3\left|\nabla^{S} v\right|^{2}-v^{3}\left(\partial_{l}\left(h_{k k}\right) w^{l}\right)+v^{2}|A|^{2}\right) \psi^{\prime}\left(v^{2}\right)+2\left|\nabla^{S} v\right|^{2} \psi^{\prime \prime}\left(v^{2}\right) .
\end{aligned}
$$

Hence

$$
\left(\frac{d}{d t}-\Delta^{S}\right) \frac{1}{2} \psi\left(v^{2}\right)=-v^{3} \psi^{\prime}\left(v^{2}\right)\left(\nabla^{S} k_{\varepsilon}, \omega\right)-\left(3\left|\nabla^{S} v\right|^{2}+v^{2}|A|^{2}\right) \psi^{\prime}\left(v^{2}\right)-2 v^{2}\left|\nabla^{S} v\right|^{2} \psi^{\prime \prime}\left(v^{2}\right) .
$$

As above, we want to conclude the proof using the weak maximum principle. So, we want to rewrite the last terms (which are gradient terms) using the gradient of $V$. Let us expand $\nabla^{S} V$.

$$
\nabla^{S} \frac{\phi^{2}|A|^{2} \psi\left(v^{2}\right)}{2}=\phi^{2}|A|^{2} \frac{1}{2} \nabla^{S} \psi\left(v^{2}\right)+|A|^{2} \psi\left(v^{2}\right) \frac{1}{2} \nabla^{S} \phi^{2}+\phi^{2} \psi\left(v^{2}\right) \frac{1}{2} \nabla^{S}|A|^{2} .
$$

So,

$$
\begin{array}{r}
\left|\nabla^{S} \frac{\phi^{2}|A|^{2} \psi\left(v^{2}\right)}{2}\right|^{2}=\phi^{4}|A|^{4} \frac{\left|\nabla^{S} \psi\left(v^{2}\right)\right|^{2}}{4}+|A|^{4} \psi^{2}\left(v^{2}\right) \frac{\left|\nabla^{S} \phi^{2}\right|^{2}}{4}+\phi^{4} \psi^{2}\left(v^{2}\right) \frac{\left.\left.\left|\nabla^{S}\right| A\right|^{2}\right|^{2}}{4} \\
+\phi^{2}|A|^{4} \psi\left(v^{2}\right)\left\langle\nabla^{S} \psi\left(v^{2}\right), \nabla^{S} \phi^{2}\right\rangle+\phi^{4}|A|^{2} \psi\left(v^{2}\right)\left\langle\nabla^{S} \psi\left(v^{2}\right), \nabla^{S}|A|^{2}\right\rangle \\
+|A|^{2} \psi^{2}\left(v^{2}\right) \phi^{2}\left\langle\nabla^{S} \phi^{2}, \nabla^{S}|A|^{2}\right\rangle .
\end{array}
$$

As a matter of fact,

$$
\begin{gathered}
\frac{1}{\phi^{2}|A|^{2} \psi\left(v^{2}\right)}\left|\nabla^{S} \frac{\phi^{2}|A|^{2} \psi\left(v^{2}\right)}{2}\right|^{2}=\phi^{2}|A|^{2} \frac{\left|\nabla^{S} \psi\left(v^{2}\right)\right|^{2}}{4 \psi\left(v^{2}\right)}+|A|^{2} \psi\left(v^{2}\right) \frac{\left|\nabla^{S} \phi^{2}\right|^{2}}{4 \phi^{2}} \\
+\phi^{2} \psi\left(v^{2}\right) \frac{\left.\left.\left|\nabla^{S}\right| A\right|^{2}\right|^{2}}{4|A|^{2}}+2|A|^{2}\left\langle\nabla^{S} \psi\left(v^{2}\right) / 2, \nabla^{S} \phi^{2} / 2\right\rangle \\
+2 \phi^{2}\left\langle\nabla^{S} \psi\left(v^{2}\right) / 2, \nabla^{S}|A|^{2} / 2\right\rangle+2 \psi\left(v^{2}\right)\left\langle\nabla^{S} \phi^{2} / 2, \nabla^{S}|A|^{2} / 2\right\rangle .
\end{gathered}
$$


We use the last equality to rewrite

$$
\begin{aligned}
\left(\frac{d}{d t}-\right. & \left.\Delta^{S}\right) \frac{\phi^{2}|A|^{2} \psi\left(v^{2}\right)}{2} \\
= & |A|^{2} \psi\left(v^{2}\right)\left(\phi\left(2 k_{\varepsilon}(\mathbf{x}, v)-\Lambda\right)-\left.\left.\left|\nabla^{S}\right| x\right|^{2}\right|^{2}\right) \\
& +\phi^{2} \psi\left(v^{2}\right)\left(-\left\langle\nabla^{S} A, \nabla^{S} A\right\rangle+|A|^{4}-2 k_{\varepsilon} g^{j s} h_{s t} g^{t l} g^{j l} h_{i j} h_{k l}-2\left\langle A,\left(\nabla^{S}\right)^{2} k_{\varepsilon}\right\rangle\right) \\
& +\phi^{2}|A|^{2}\left(-v^{3} \psi^{\prime}\left(v^{2}\right)\left(\nabla^{S} k_{\varepsilon}, \omega\right)-\left(3\left|\nabla^{S} v\right|^{2}+v^{2}|A|^{2}\right) \psi^{\prime}\left(v^{2}\right)-2 v^{2}\left|\nabla^{S} v\right|^{2} \psi^{\prime \prime}\left(v^{2}\right)\right) \\
& -\frac{1}{\phi^{2}|A|^{2} \psi\left(v^{2}\right)}\left|\nabla^{S} \frac{\phi^{2}|A|^{2} \psi\left(v^{2}\right)}{2}\right|^{2} \\
& +\phi^{2}|A|^{2} \frac{\left|\nabla^{S} \psi\left(v^{2}\right)\right|^{2}}{4 \psi\left(v^{2}\right)}+|A|^{2} \psi\left(v^{2}\right) \frac{\left|\nabla^{S} \phi^{2}\right|^{2}}{4 \phi^{2}}+\phi^{2} \psi\left(v^{2}\right) \frac{\left.\left.\left|\nabla^{S}\right| A\right|^{2}\right|^{2}}{4|A|^{2}} .
\end{aligned}
$$

Let us rewrite some terms as follows:

$$
\begin{aligned}
\left|\nabla^{S} \phi^{2}\right|^{2} & =4 \phi^{2} \cdot\left|-2 \mathbf{x}^{T}\right|^{2}=4 \phi^{2}\left(4|\mathbf{x}|^{2}-4(\mathbf{x}, v)\right), \\
\left|\nabla^{S} \psi\left(v^{2}\right)\right|^{2} & =\psi^{\prime}\left(v^{2}\right)^{2}\left|\nabla^{S} v^{2}\right|^{2}=4 \psi^{\prime}\left(v^{2}\right)^{2} v^{6} \sum_{k}\left(\lambda_{k} \omega^{k}\right)^{2}, \\
\left.\left.\left|\nabla^{S}\right| A\right|^{2}\right|^{2} & =4 \sum_{i}\left(\partial_{i}\left(h_{l l}\right) \lambda_{l}\right)^{2}, \\
\left|\nabla^{S} A\right|^{2} & =\sum_{i, k, l}\left(\partial_{i}\left(h_{k m}\right)\right)^{2} .
\end{aligned}
$$

In addition, we have the obvious estimate

$$
\left.\left.\left|\nabla^{S}\right| A\right|^{2}\right|^{2} \leqslant 4|A|^{2}\left|\nabla^{S} A\right|^{2} .
$$

So,

$$
\begin{aligned}
\phi^{2}|A|^{2} & \frac{\left|\nabla^{S} \psi\left(v^{2}\right)\right|^{2}}{4 \psi\left(v^{2}\right)}+|A|^{2} \psi\left(v^{2}\right) \frac{\left|\nabla^{S} \phi^{2}\right|^{2}}{4 \phi^{2}}+\phi^{2} \psi\left(v^{2}\right) \frac{\left.\left.\left|\nabla^{S}\right| A\right|^{2}\right|^{2}}{4|A|^{2}} \\
& \leqslant \phi^{2}|A|^{2} \frac{\psi^{\prime}\left(v^{2}\right)^{2} v^{6} \sum_{k}\left(\lambda_{k} \omega^{k}\right)^{2}}{\psi\left(v^{2}\right)}+4|A|^{2} \psi\left(v^{2}\right)\left(|\mathbf{x}|^{2}-(\mathbf{x}, v)^{2}\right)+\phi^{2} \psi\left(v^{2}\right)\left|\nabla^{S} A\right|^{2} .
\end{aligned}
$$

We plug this inequality into (25) and obtain

$$
\begin{aligned}
\left(\frac{d}{d t}\right. & \left.-\Delta^{S}\right) \frac{\phi^{2}|A|^{2} \psi\left(v^{2}\right)}{2} \leqslant|A|^{2} \psi\left(v^{2}\right)\left(\phi\left(2 k_{\varepsilon}(\mathbf{x}, v)-\Lambda\right)-\left.\left.\left|\nabla^{S}\right| x\right|^{2}\right|^{2}\right) \\
& +\phi^{2} \psi\left(v^{2}\right)\left(-\left\langle\nabla^{S} A, \nabla^{S} A\right\rangle+|A|^{4}-2 k_{\varepsilon} g^{j s} h_{s t} g^{t l} g^{j l} h_{i j} h_{k l}-2\left\langle A,\left(\nabla^{S}\right)^{2} k_{\varepsilon}\right\rangle\right) \\
& +\phi^{2}|A|^{2}\left(-v^{3} \psi^{\prime}\left(v^{2}\right)\left(\nabla^{S} k_{\varepsilon}, \omega\right)-\left(3\left|\nabla^{S} v\right|^{2}+v^{2}|A|^{2}\right) \psi^{\prime}\left(v^{2}\right)-2 v^{2}\left|\nabla^{S} v\right|^{2} \psi^{\prime \prime}\left(v^{2}\right)\right) \\
& -\frac{1}{\phi^{2}|A|^{2} \psi\left(v^{2}\right)}\left|\nabla \frac{\phi^{2}|A|^{2} \psi\left(v^{2}\right)}{2}\right|^{2} \\
& +\phi^{2}|A|^{2} \frac{\psi^{\prime}\left(v^{2}\right)^{2} v^{6} \sum_{k}\left(\lambda_{k} \omega^{k}\right)^{2}}{\psi\left(v^{2}\right)}+4|A|^{2} \psi\left(v^{2}\right)\left(|\mathbf{x}|^{2}-(\mathbf{x}, v)^{2}\right)+\phi^{2} \psi\left(v^{2}\right)\left|\nabla^{S} A\right|^{2}
\end{aligned}
$$


Let us regroup some terms (noting that $\left|\nabla^{S} v\right|^{2}=v^{4} \sum_{i}\left(\lambda_{i} \omega^{i}\right)^{2}$ ), we get

$$
\begin{aligned}
\left(\frac{d}{d t}\right. & \left.-\Delta^{S}\right) \frac{\phi^{2}|A|^{2} \psi\left(v^{2}\right)}{2} \\
& \leqslant|A|^{2} \psi\left(v^{2}\right)\left(\phi\left(2 k_{\varepsilon}(\mathbf{x}, v)-\Lambda\right)\right) \\
& +\phi^{2}|A|^{4}\left(\psi\left(v^{2}\right)-v^{2} \psi^{\prime}\left(v^{2}\right)\right)-2 \phi^{2} \psi\left(v^{2}\right) k_{\varepsilon} g^{j s} h_{s t} g^{t l} g^{j l} h_{i j} h_{k l}-2 \phi^{2} \psi\left(v^{2}\right)\left\langle A,\left(\nabla^{S}\right)^{2} k_{\varepsilon}\right\rangle \\
& -\phi^{2}|A|^{2} v^{3} \psi^{\prime}\left(v^{2}\right)\left(\nabla^{S} k_{\varepsilon}, \omega\right)-\frac{1}{\phi^{2}|A|^{2} \psi\left(v^{2}\right)}\left|\nabla^{S} \frac{\phi^{2}|A|^{2} \psi\left(v^{2}\right)}{2}\right|^{2} \\
& +\phi^{2}|A|^{2} \sum_{i}\left(\lambda_{i} \omega^{i}\right)^{2}\left(\frac{v^{6} \psi^{\prime}\left(v^{2}\right)^{2}}{\psi\left(v^{2}\right)}-3 v^{4} \psi^{\prime}\left(v^{2}\right)-2 v^{6} \psi^{\prime \prime}\left(v^{2}\right)\right) .
\end{aligned}
$$

Then, we note that

$$
\frac{v^{6} \psi^{\prime}\left(v^{2}\right)^{2}}{\psi\left(v^{2}\right)}-3 v^{4} \psi^{\prime}\left(v^{2}\right)-2 v^{6} \psi^{\prime \prime}\left(v^{2}\right)=-\frac{2 v^{4}+\gamma v^{6}}{\left(1-\gamma v^{2}\right)^{3}} \leqslant 0
$$

and

$$
\psi\left(v^{2}\right)-v^{2} \psi^{\prime}\left(v^{2}\right)=-\gamma \psi^{2}\left(v^{2}\right) \leqslant 0 .
$$

So,

$$
\begin{aligned}
\left(\frac{d}{d t}-\Delta^{S}\right) \frac{\phi^{2}|A|^{2} \psi\left(v^{2}\right)}{2} & \leqslant \phi^{2} \psi\left(v^{2}\right)\left(-\gamma|A|^{4}-2 k_{\varepsilon} \sum_{i} \lambda_{i}^{3}-2\left\langle A,\left(\nabla^{S}\right)^{2} k_{\varepsilon}\right)\right) \\
& -\phi^{2}|A|^{2} v^{3} \psi^{\prime}\left(v^{2}\right)\left(\nabla^{S} k_{\varepsilon}, \omega\right)-\phi^{2}|A|^{2} \sum_{i}\left(\lambda_{i} \omega^{i}\right)^{2} \frac{2 v^{4}+\gamma v^{6}}{\left(1-\gamma v^{2}\right)^{3}},
\end{aligned}
$$

what was expected.

We now show that $M_{t}$ can be locally written as a Lipschitz graph, with Lipschitz constant independent of $\varepsilon$.

Proposition 5 Let $\varepsilon \leqslant r$. Then, for every $t \in\left[0, \min \left(T_{\varepsilon}, T_{1}\right)\right), M_{t} \cap B_{i}$ can be written as a Lipschitz graph over $\omega_{i}^{\perp}$, with Lipschitz constant independent of $\varepsilon$.

Proof. We want to show that the quantity $\left(v, \omega_{i}\right)$ is bounded from below, or, equivalently, that $v:=\left(v, \omega_{i}\right)^{-1}$ is bounded from above on every ball $B_{i}$. We want to estimate the quantity $v^{2} \phi^{2}$ (we drop the explicit dependence on the index $i$ ) using Lemma 4.

We choose $\Lambda$ such that the last term in (22) is nonpositive (take for instance $\Lambda=2 N R$ ). We also have to control

$$
v\left(\nabla^{S} k_{\varepsilon}, \omega\right)=(v, \omega)^{-1}\left(\left(\nabla k_{\varepsilon}, \omega\right)-\left(\nabla k_{\varepsilon}, v\right)(v, \omega)\right)=(v, \omega)^{-1}\left(\nabla k_{\varepsilon}, \omega\right)-\left(\nabla k_{\varepsilon}, v\right) .
$$

Proposition 4 provides immediately

$$
(v, \omega)^{-1}\left(\nabla k_{\varepsilon}, \omega\right)-\left(\nabla k_{\varepsilon}, v\right) \geqslant(v, \omega)^{-1} \frac{\left|\nabla k_{\varepsilon}\right|}{2}-\left|\nabla k_{\varepsilon}\right|
$$

which is nonnegative as soon as $(\omega, v) \leqslant \frac{1}{2}$. From Lemma 4 and the weak maximum principle (see [22]), we obtain that $\left\|v^{2} \phi^{2}\right\|_{\infty}(t) \leqslant \max \left(\left\|v^{2} \phi^{2}\right\|_{\infty}(0), 4 R^{2}\right)$. Thanks to Lemma 3 , this provides a uniform Lipschitz bound on the whole $M_{t}$, for $t \leqslant T_{1}$. 
Recalling Theorem 8.1 in [15], from Proposition 5 it follows that, if $T_{\varepsilon}<T_{1}$, the second fundamental form of $M_{t}$ blows up as $t \rightarrow T_{\varepsilon}$. Let us show that it does not happen.

PRoposition 6 For every $\varepsilon \leqslant r$, there exists $C_{\varepsilon}>0$ such that

$$
\|A\|_{L^{\infty}\left(M_{t}\right)} \leqslant C_{\varepsilon} \quad \text { for all } t \in\left[0, \min \left(T_{\varepsilon}, T_{1}\right)\right) .
$$

Proof. As in [9], we are interested in the evolution of the quantity

$$
\frac{\phi^{2}|A|^{2} \psi\left(v^{2}\right)}{2}
$$

and use the estimates of Lemma 5. Notice that

$$
\left|\lambda_{i}\right|^{3}=\left|\lambda_{i}\right|\left|\lambda_{i}\right|^{2} \leqslant \frac{1}{2 \alpha} \lambda_{i}^{4}+\frac{\alpha}{2} \lambda_{i}^{2} .
$$

Choosing $\alpha$ such that $\frac{2 N}{\alpha} \leqslant \frac{\gamma}{2}$, one can write

$$
\left|-2 k_{\varepsilon} \phi^{2} \psi\left(v^{2}\right) \sum_{i} \lambda_{i}^{3}\right| \leqslant \phi^{2} \psi\left(v^{2}\right)\left(\frac{\gamma}{2}|A|^{4}+N \alpha|A|^{2}\right) .
$$

In addition, as soon as $|A|^{2} \geqslant 1$, one has $\left\langle A,\left(\nabla^{S}\right)^{2} k_{\varepsilon}\right\rangle \leqslant|A|^{2}\left|\nabla^{2} k_{\varepsilon}\right|$. One can also notice that as above, $v\left(\nabla^{S} k_{\varepsilon}, \omega\right) \geqslant 0$ as soon as $v \geqslant 2$. On the other hand, if $v \leqslant 2$, one has $v^{3} \psi^{\prime}\left(v^{2}\right)=$ $\frac{\psi(v) v}{1-\gamma v^{2}} \leqslant 4 \psi(v)$ for $\gamma$ sufficiently small.

So, anyway, if $|A| \geqslant 1$,

$$
\begin{aligned}
\left(\frac{d}{d t}-\Delta^{S}\right) \frac{\phi^{2}|A|^{2} \psi\left(v^{2}\right)}{2} \leqslant 2 N \alpha \frac{\phi^{2}|A|^{2} \psi\left(v^{2}\right)}{2}+4\left|\nabla^{2} k_{\varepsilon}\right| \frac{\phi^{2}|A|^{2} \psi\left(v^{2}\right)}{2} & \\
& +8 \frac{\phi^{2}|A|^{2} \psi\left(v^{2}\right)}{2}\left|\nabla^{S} k_{\varepsilon}\right| .
\end{aligned}
$$

Finally, we apply the maximum principle to the quantity

$$
\tilde{A}:=e^{-\left(2 N \alpha+4\left\|\nabla^{2} k_{\varepsilon}\right\|_{\infty}+8\left\|\nabla k_{\varepsilon}\right\|_{\infty}\right) t} \frac{\phi^{2}|A|^{2} \psi\left(v^{2}\right)}{2}
$$

which satisfies

$$
\left(\frac{d}{d t}-\Delta^{S}\right) \tilde{A} \leqslant 0
$$

It provides

$$
\forall t \leqslant \min \left(T_{\varepsilon}, T_{1}\right), \quad\|\tilde{A}\|_{\infty}(t) \leqslant\|\tilde{A}\|_{\infty}(0)
$$

which shows that $\frac{\phi^{2}|A|^{2} \psi\left(v^{2}\right)}{2}$ does not blow up.

Using Lemma 3 and choosing $\gamma$ such that $\psi\left(v^{2}\right)$ is bounded and remains far from zero, we know that $|A|$ does not blow up for $t \leqslant T_{1}$.

COROLlary 1 There exists $T_{1}$, depending only on the dimension, $\|k\|_{\infty}$ and the radius in the ball condition for $M_{0}$, such that there exists a solution $M_{t}^{\varepsilon}$ of the mean curvature flow with forcing term $k_{\varepsilon}$ on $\left[0, T_{1}\right)$. 
The surfaces $M_{t}^{\varepsilon}$ are uniformly Lipschitz and every $M_{t}^{\varepsilon} \cap B_{i}$ can be written as the graph of some function $u_{i}^{\varepsilon}(x, t)$. All the $u_{i}^{\varepsilon}$ are Lipchitz (in space) with a constant which depends neither on $i$ nor in $\varepsilon$. We want to show that they are also equicontinuous in time.

PROPOSITION 7 The functions $u_{i}^{\varepsilon}$ are Lipschitz continuous in $x$ and 1/2-Hölder continuous in $t$ on $B_{i} \times\left[0, T_{1}\right)$, uniformly with respect to $\varepsilon$ and $i$.

In addition, they are (classical) solutions of the equation

$$
\partial_{t} u_{i}^{\varepsilon}=\sqrt{1+\left|\nabla u_{i}^{\varepsilon}\right|^{2}} \operatorname{div}\left(\frac{\nabla u_{i}^{\varepsilon}}{\sqrt{1+\left|\nabla u_{i}^{\varepsilon}\right|^{2}}}\right)-\sqrt{1+\left|\nabla u_{i}^{\varepsilon}\right|^{2}} k_{\varepsilon}\left(x, u_{i}^{\varepsilon}\right) .
$$

Proof. Let $\delta$ be fixed (we drop the index $\varepsilon$ in what follows), and let $t_{0} \in\left[0, T_{1}\right)$. Let $x_{0} \in M_{t}$ and $i$ such that $x_{0} \in B_{i}$. Then, $\left(v\left(x_{0}\right), \omega_{i}\right)^{-1}$ is bounded above and $M_{t}$ is the graph of a function $u$ over $\omega_{i}^{\perp}$. Then, let $x_{1}=x_{0}+\delta \omega_{i}$. Thanks to the Lipschitz condition, there is a ball $B_{1 / C \delta}\left(x_{1}\right)$ that does not touch $M_{t}$. Evolving by mean curvature with forcing term $k_{\varepsilon}$, this ball vanishes in a positive time $T_{\delta} \geqslant \omega(\delta):=\frac{\delta^{2}}{C^{2}(2 d+1)}$ (note that $T_{\delta}$ does not depend on $\varepsilon$ ). By comparison principle, for $t \in\left[t_{0}, t_{0}+\omega(\delta)\right), M_{t}$ does not go beyond $x_{1}$. That is equivalent to say that $u$ is $1 / 2$-Hölder continuous in time, with a constant independent of $\varepsilon$.

The equation satisfied by $u_{i}^{\varepsilon}$ is usual. One just has to notice that with the definitions above,

$$
\operatorname{div}\left(\frac{\nabla u_{i}^{\varepsilon}}{\sqrt{1+\left|\nabla u_{i}^{\varepsilon}\right|^{2}}}\right)=-H
$$

We now pass to the limit as $\varepsilon$ goes to zero. By Proposition 7 , the family $\left(u_{i}^{\varepsilon}\right)$ is equi-Lipschitz in space and equi-continuous in time on $B_{i} \times\left[0, T_{1}\right)$. Therefore, by Arzelà-Ascoli's Theorem one can find a sequence $\varepsilon_{n} \rightarrow 0$ and continuous functions $u_{i}$ such that, for every $i, u_{i}^{\varepsilon_{n}} \underset{n \rightarrow \infty}{\longrightarrow} u_{i}$ locally uniformly on $B_{i} \times\left[0, T_{1}\right)$.

Proposition 8 The functions $u_{i}$ are viscosity solutions of (3) on $B_{i} \times\left[0, T_{1}\right.$ ), with obstacles $U \cap B_{i}$ (see Appendix 5).

Proof. Thanks to Proposition 4, every $x \in B_{i}$ can be decomposed as $x=x^{\prime}+z \omega_{i}$ with $z=$ $\left(x, \omega_{i}\right)$. Then, there exists functions $\psi_{i}^{ \pm}$of class $C^{1,1}$ such that

$$
U \cap B_{i}=\left\{\left(x^{\prime}, z\right) \in B_{i}: \psi_{i}^{-}\left(x^{\prime}\right) \leqslant z \leqslant \psi_{i}^{+}\left(x^{\prime}\right)\right\}
$$

For simplicity we shall drop the explicit dependence on the index $i$. Since $u^{\varepsilon}(x, 0)=u_{0}(x)$ for all $\varepsilon$, and $u^{\varepsilon_{n}}$ converges uniformly to $u$ as $n \rightarrow+\infty$, it is clear that $u(x, 0)=u_{0}(x)$.

Condition (A3) immediately follows from Proposition 3.

We now check that $u$ is a subsolution of (3). Let $\left(x_{0}, t_{0}\right) \in \mathbb{R}^{d} \times \mathbb{R}$ and $\varphi \in C^{2}$ such that $\psi^{-}\left(x_{0}, t_{0}\right)<u\left(x_{0}, t_{0}\right)$ and

$$
(u-\varphi)\left(x_{0}, t_{0}\right)=\max _{\left|(x, t)-\left(x_{0}, t_{0}\right)\right| \leqslant r}(u-\varphi)(x, t) .
$$

One can change $\varphi$ so that $\left(x_{0}, t_{0}\right)$ is a strict maximum point, and $u\left(x_{0}, t_{0}\right)=\varphi\left(x_{0}, t_{0}\right)$. Let $2 \delta:=$ $u\left(x_{0}, t_{0}\right)-\psi^{-}\left(x_{0}, t_{0}\right)$. Thanks to the definition of $k_{\varepsilon}$, for all $\varepsilon \leqslant \delta$, we have $k_{\varepsilon}(x, \varphi(x, t)) \geqslant 0$ in 
a small neighborhood $V$ of $\left(x_{0}, t_{0}\right)$. Hence, for $\varepsilon$ sufficiently small $u^{\varepsilon}-\varphi$ attains its maximum in $V$ at $\left(x_{\varepsilon}, t_{\varepsilon}\right)$, with $\left(x_{\varepsilon}, t_{\varepsilon}\right) \rightarrow\left(x_{0}, t_{0}\right)$ as $\varepsilon \rightarrow 0$. Since $u^{\varepsilon}$ is a classical solution of (26), it is also a viscosity solution, therefore

$$
\varphi_{t}-\sqrt{1+|\nabla \varphi|^{2}} \operatorname{div}\left(\frac{\nabla \varphi}{\sqrt{1+|\nabla \varphi|^{2}}}\right) \leqslant-\sqrt{1+|\nabla \varphi|^{2}} k_{\varepsilon}(x, \varphi) \leqslant 0 \quad \operatorname{at}\left(x_{\varepsilon}, t_{\varepsilon}\right) .
$$

Letting $\varepsilon \rightarrow 0$ we obtain that $u$ is a subsolution of (3). A similar argument shows that $u$ is also a supersolution of (3), and this concludes the proof.

Conclusion of the proof of Theorem 1. The result in [21, Theorem 4.1] (see also Section A.4) applies, showing that the functions $u_{i}$ are of class $C^{1,1}$. As the uniform convergence $u_{i}^{\varepsilon_{n}}$ implies the Hausdorff convergence of $M_{t}^{\varepsilon_{n}}$ to a limit $M_{t}$ such that $M_{t} \cap B_{i}=\operatorname{graph}\left(u_{i}(t)\right)$, we built a $C^{1,1}$ evolution to the mean curvature motion with obstacles on the time interval $\left[0, T_{1}\right)$. Thanks to [1, Theorem 4.8 and Corollary 4.9] this evolution is also unique. This concludes the proof of Theorem 1.

\section{Proof of Theorem 2}

Let $\psi_{\varepsilon}^{ \pm}$be smooth functions such that $\psi_{\varepsilon}^{ \pm} \rightarrow \psi^{ \pm}$as $\varepsilon \rightarrow 0$, uniformly in $C^{1,1}\left(\mathbb{R}^{d}\right)$, and let $N>0$ be such that

$$
N \geqslant\left\|\sqrt{1+\left|\psi_{\varepsilon}^{ \pm}\right|^{2}} \operatorname{div}\left(\frac{\psi_{\varepsilon}^{ \pm}}{\sqrt{1+\left|\psi_{\varepsilon}^{ \pm}\right|^{2}}}\right)\right\|_{L^{\infty}\left(\mathbb{R}^{d}\right)} \quad \text { for all } \varepsilon>0 .
$$

We proceed as in Section 3 and we approximate (3), (4) with the forced mean curvature equation

$$
u_{t}=\sqrt{1+|\nabla u|^{2}}\left[\operatorname{div}\left(\frac{\nabla u}{\sqrt{1+|\nabla u|^{2}}}\right)+k_{\varepsilon}(x, u)\right],
$$

where

$$
k_{\varepsilon}(x, u)=2 N\left(\chi\left(\frac{\psi_{\varepsilon}^{-}(x)-u}{\varepsilon}\right)-\chi\left(\frac{u-\psi_{\varepsilon}^{+}(x)}{\varepsilon}\right)\right),
$$

and $\chi$ is a smooth increasing function such that $\chi(s) \equiv 0$ for all $s \in(-\infty, 0]$, and $\chi(s) \equiv 1$ for all $s \in[1, \infty)$. In particular $\partial_{u} k_{\varepsilon}(x, u) \leqslant 0$ for all $(x, u)$.

Note that the signs between (28) and (5) are reversed.

Notice that $k_{\varepsilon} \rightarrow k$ as $\varepsilon \rightarrow 0$, with

$$
k(x, u)=\left\{\begin{array}{c}
2 N \text { if } u<\psi^{-}(x) \\
-2 N \text { if } u>\psi^{+}(x) . \\
0 \text { elsewhere }
\end{array}\right.
$$

We denote by $u_{\varepsilon}$ the solution of the approximate problem (28), which exists and is smooth for short times.

Proposition 9 The solution $u_{\varepsilon}$ is defined for $t \in[0,+\infty)$, and satisfies the estimates

$$
\begin{array}{lll}
\left\|u_{\varepsilon}(\cdot, t)\right\|_{W^{1, \infty}\left(\mathbb{R}^{d}\right)} \leqslant C & & \text { for all } t \in[0,+\infty), \\
\left\|u_{\varepsilon}(\cdot, t)\right\|_{W^{2, \infty}\left(\mathbb{R}^{d}\right)} \leqslant C(T) & & \text { for all } t \in[0, T] .
\end{array}
$$


Proof. Estimate (29) follows from Proposition 5, choosing $B_{i}=\mathbb{R}^{d+1}, \omega_{i}=e_{d+1}$ and $\phi \equiv 1$. Estimate (30) follows from (29) and Proposition 6.

In what follows, we use intrinsic derivatives on the graph $M_{t}:=\left\{\left(x, u_{\varepsilon}(x, t)\right)\right\}$, which will be denoted as above by an exponent $S$. The metric on $M_{t}$ is

$$
g_{i j}=\delta_{i j}+\partial_{i} u_{\varepsilon} \partial_{j} u
$$

with inverse

$$
g^{i j}=\delta_{i j}-\frac{\partial_{i} u_{\varepsilon} \partial_{j} u_{\varepsilon}}{1+\left|\nabla u_{\varepsilon}\right|^{2}} .
$$

The tangential gradient of a function $f$ defined on $M_{t}$ is given by

$$
\left(\nabla^{S} f\right)^{i}=g^{i j} \partial_{j} f=\partial_{i} f-\frac{\partial_{i} u_{\varepsilon} \partial_{j} u_{\varepsilon}}{1+\left|\nabla u_{\varepsilon}\right|^{2}} \partial_{j} f
$$

so that

$$
\left(\nabla^{S} f, \nabla u_{\varepsilon}\right)=\left(\nabla f, \nabla u_{\varepsilon}\right)-\frac{\left|\nabla u_{\varepsilon}\right|^{2}}{1+\left|\nabla u_{\varepsilon}\right|^{2}}\left(\nabla f, \nabla u_{\varepsilon}\right)=\frac{1}{1+\left|\nabla u_{\varepsilon}\right|^{2}}\left(\nabla f, \nabla u_{\varepsilon}\right),
$$

and

$$
\begin{aligned}
\left|\nabla^{S} f\right|^{2} & =\left(f_{i}-\left(u_{\varepsilon}\right)_{i} \sum_{j} \frac{\left(u_{\varepsilon}\right)_{j} f_{j}}{1+\left|\nabla u_{\varepsilon}\right|^{2}}\right)^{2} \\
& =|\nabla f|^{2}+\left(u_{\varepsilon}\right)_{i}^{2}\left(\frac{\left(\nabla u_{\varepsilon}, \nabla f\right)}{1+\left|\nabla u_{\varepsilon}\right|^{2}}\right)^{2}-2 \frac{\left(u_{\varepsilon}\right)_{i}\left(u_{\varepsilon}\right)_{j} f_{i} f_{j}}{1+\left|\nabla u_{\varepsilon}\right|^{2}} \\
& =|\nabla f|^{2}+\frac{\left|\nabla u_{\varepsilon}\right|^{2}}{1+\left|\nabla u_{\varepsilon}\right|^{2}} \frac{\left(\nabla u_{\varepsilon}, \nabla f\right)^{2}}{1+\left|\nabla u_{\varepsilon}\right|^{2}}-2 \frac{\left(\nabla u_{\varepsilon}, \nabla f\right)^{2}}{1+\left|\nabla u_{\varepsilon}\right|^{2}} \\
& =|\nabla f|^{2}-\frac{\left(\nabla u_{\varepsilon}, \nabla f\right)^{2}}{1+\left|\nabla u_{\varepsilon}\right|^{2}}-\frac{\left(\nabla u_{\varepsilon}, \nabla f\right)^{2}}{\left(1+\left|\nabla u_{\varepsilon}\right|^{2}\right)^{2}} .
\end{aligned}
$$

In addition, the Laplace-Beltrami operator applied to $f$ is

$$
\Delta^{S} f=g^{i j} f_{i j}=\Delta f-\frac{\partial_{i} u_{\varepsilon} \partial_{j} u_{\varepsilon}}{1+\left|\nabla u_{\varepsilon}\right|^{2}} f_{i j}=\Delta f-\frac{\left(\nabla u_{\varepsilon} \nabla^{2} f, \nabla u_{\varepsilon}\right)}{1+\left|\nabla u_{\varepsilon}\right|^{2}} .
$$

Proposition 10 The quantity $\left\|\left(u_{\varepsilon}\right)_{t}^{2}\right\|_{\infty}(t)$ is nonincreasing in time. In particular,

$$
\left\|\left(u_{\varepsilon}\right)_{t}(\cdot, t)\right\|_{L^{\infty}\left(\mathbb{R}^{d}\right)} \leqslant\left\|\sqrt{1+\left|\nabla u_{0}\right|^{2}} \operatorname{div}\left(\frac{\nabla u_{0}}{\sqrt{1+\left|\nabla u_{0}\right|^{2}}}\right)\right\|_{L^{\infty}\left(\mathbb{R}^{d}\right)} .
$$

Proof. We compute

$$
\frac{d}{d t} \frac{\left(u_{\varepsilon}\right)_{t}^{2}}{2}=\left(u_{\varepsilon}\right)_{t}\left[\sqrt{1+\left|\nabla u_{\varepsilon}\right|^{2}}\left(\operatorname{div}\left(\frac{\nabla u_{\varepsilon}}{\sqrt{1+\left|\nabla u_{\varepsilon}\right|^{2}}}\right)+k_{\varepsilon}\right)\right]_{t} .
$$


Expanding this expression, we get

$$
\begin{aligned}
\frac{d}{d t} \frac{\left(u_{\varepsilon}\right)_{t}^{2}}{2}= & \left(u_{\varepsilon}\right)_{t}\left[\frac{\left(\nabla\left(u_{\varepsilon}\right)_{t}, \nabla u_{\varepsilon}\right)}{\sqrt{1+\left|\nabla u_{\varepsilon}\right|^{2}}}\left(\operatorname{div}\left(\frac{\nabla u_{\varepsilon}}{\sqrt{1+\left|\nabla u_{\varepsilon}\right|^{2}}}\right)+k_{\varepsilon}\right)\right. \\
& \left.+\sqrt{1+\left|\nabla u_{\varepsilon}\right|^{2}}\left(\operatorname{div}\left(\frac{\left(\nabla u_{\varepsilon}\right)_{t}}{\sqrt{1+\left|\nabla u_{\varepsilon}\right|^{2}}}-\frac{\left(\left(\nabla u_{\varepsilon}\right)_{t}, \nabla u_{\varepsilon}\right) \nabla u_{\varepsilon}}{\left(1+\left|\nabla u_{\varepsilon}\right|^{2}\right)^{3 / 2}}\right)+\left(u_{\varepsilon}\right)_{t} \partial_{u} k_{\varepsilon}\right)\right] .
\end{aligned}
$$

Let us compute more explicitly the three terms of the expression above:

$$
\begin{aligned}
& \left(u_{\varepsilon}\right)_{t} \frac{\left(\left(\nabla u_{\varepsilon}\right)_{t}, \nabla u_{\varepsilon}\right)}{\sqrt{1+\left|\nabla u_{\varepsilon}\right|^{2}}}\left(\operatorname{div}\left(\frac{\nabla u_{\varepsilon}}{\sqrt{1+\left|\nabla u_{\varepsilon}\right|^{2}}}\right)+k_{\varepsilon}\right) \\
& =\frac{\left(\nabla\left(\frac{\left(u_{\varepsilon}\right)_{t}^{2}}{2}\right), \nabla u_{\varepsilon}\right)}{\sqrt{1+\left|\nabla u_{\varepsilon}\right|^{2}}}\left(\frac{\Delta u}{\sqrt{1+\left|\nabla u_{\varepsilon}\right|^{2}}}-\frac{\left(u_{\varepsilon}\right)_{i}\left(\nabla u_{\varepsilon},\left(\nabla u_{\varepsilon}\right)_{i}\right)}{\left(1+\left|\nabla u_{\varepsilon}\right|^{2}\right)^{3 / 2}}+k_{\varepsilon}\right) \\
& =\left(\nabla\left(\frac{\left(u_{\varepsilon}\right)_{t}^{2}}{2}\right), \nabla u_{\varepsilon}\right)\left(\frac{\Delta u_{\varepsilon}}{1+\left|\nabla u_{\varepsilon}\right|^{2}}-\frac{\left(\nabla u_{\varepsilon}, \nabla\left(\frac{\left|\nabla u_{\varepsilon}\right|^{2}}{2}\right)\right)}{\left(1+\left|\nabla u_{\varepsilon}\right|^{2}\right)^{2}}+k_{\varepsilon}\right) \text {, } \\
& \left(u_{\varepsilon}\right)_{t} \operatorname{div}\left(\frac{\nabla\left(u_{\varepsilon}\right)_{t}}{\sqrt{1+\left|\nabla u_{\varepsilon}\right|^{2}}}\right)=\left(u_{\varepsilon}\right)_{t} \partial_{i}\left(\frac{\left(u_{\varepsilon}\right)_{t i}}{\sqrt{1+\left|\nabla u_{\varepsilon}\right|^{2}}}\right) \\
& =\frac{\left(u_{\varepsilon}\right)_{t}\left(u_{\varepsilon}\right)_{t i i}}{\sqrt{1+\left|\nabla u_{\varepsilon}\right|^{2}}}-\frac{1}{\left(1+\left|\nabla u_{\varepsilon}\right|^{2}\right)^{3 / 2}}\left(u_{\varepsilon}\right)_{t}\left(u_{\varepsilon}\right)_{t i}\left(\nabla u_{\varepsilon},\left(\nabla u_{\varepsilon}\right)_{i}\right) \\
& =\frac{\left(u_{\varepsilon}\right)_{t} \Delta\left(u_{\varepsilon}\right)_{t}}{\sqrt{1+\nabla u_{\varepsilon}^{2}}}-\frac{1}{\left(1+\left|\nabla u_{\varepsilon}\right|^{2}\right)^{3 / 2}}\left(u_{\varepsilon}\right)_{t}\left(u_{\varepsilon}\right)_{t i} \partial_{i}\left(\frac{\left|\nabla u_{\varepsilon}\right|^{2}}{2}\right) \\
& =\frac{\left(u_{\varepsilon}\right)_{t} \Delta\left(u_{\varepsilon}\right)_{t}}{\sqrt{1+\nabla u_{\varepsilon}^{2}}}-\frac{1}{\left(1+\left|\nabla u_{\varepsilon}\right|^{2}\right)^{3 / 2}}\left(\nabla\left(\frac{\left(u_{\varepsilon}\right)_{t}^{2}}{2}\right), \nabla\left(\frac{\left|\nabla u_{\varepsilon}\right|^{2}}{2}\right)\right)
\end{aligned}
$$

and

$$
\begin{aligned}
\left(u_{\varepsilon}\right)_{t} \operatorname{div} & \left(\frac{\left(\left(\nabla u_{\varepsilon}\right)_{t}, \nabla u_{\varepsilon}\right) \nabla u_{\varepsilon}}{\left(1+\left|\nabla u_{\varepsilon}\right|^{2}\right)^{3 / 2}}\right) \\
& =\Delta u_{\varepsilon} \frac{\left(\nabla u_{\varepsilon},\left(u_{\varepsilon}\right)_{t} \nabla\left(u_{\varepsilon}\right)_{t}\right)}{\left(1+\left|\nabla u_{\varepsilon}\right|^{2}\right)^{3 / 2}}+\frac{\left(u_{\varepsilon}\right)_{t}\left(u_{\varepsilon}\right)_{t i j}\left(u_{\varepsilon}\right)_{j}\left(u_{\varepsilon}\right)_{i}}{\left(1+\left|\nabla u_{\varepsilon}\right|^{2}\right)^{3 / 2}}+\frac{\left(\left(u_{\varepsilon}\right)_{i} \nabla\left(u_{\varepsilon}\right)_{i},\left(u_{\varepsilon}\right)_{t} \nabla\left(u_{\varepsilon}\right)_{t}\right)}{\left(1+\left|\nabla u_{\varepsilon}\right|^{2}\right)^{3 / 2}} \\
& -3\left(u_{\varepsilon}\right)_{i} \frac{\left(\left(u_{\varepsilon}\right)_{t} \nabla\left(u_{\varepsilon}\right)_{t}, \nabla u_{\varepsilon}\right)\left(\nabla\left(u_{\varepsilon}\right)_{i}, \nabla u_{\varepsilon}\right)}{\left(1+\left|\nabla u_{\varepsilon}\right|^{2}\right)^{5 / 2}} \\
& =\Delta u_{\varepsilon} \frac{\left(\nabla u_{\varepsilon}, \nabla\left(\frac{\left(u_{\varepsilon}\right)_{t}^{2}}{2}\right)\right)}{\left(1+\left|\nabla u_{\varepsilon}\right|^{2}\right)^{3 / 2}}+\frac{\left(u_{\varepsilon}\right)_{t}\left(u_{\varepsilon}\right)_{t i j}\left(u_{\varepsilon}\right)_{j}\left(u_{\varepsilon}\right)_{i}}{\left(1+\left|\nabla u_{\varepsilon}\right|^{2}\right)^{3 / 2}}+\frac{\left(\nabla\left(\frac{\left|\nabla u_{\varepsilon}\right|^{2}}{2}\right), \nabla\left(\frac{\left(u_{\varepsilon}\right)_{t}^{2}}{2}\right)\right)}{\left(1+\left|\nabla u_{\varepsilon}\right|^{2}\right)^{3 / 2}} \\
& -3 \frac{\left(\nabla\left(\frac{\left(u_{\varepsilon}\right)_{t}^{2}}{2}\right), \nabla u_{\varepsilon}\right)\left(\nabla\left(\frac{\left|\nabla u_{\varepsilon}\right|^{2}}{2}\right), \nabla u_{\varepsilon}\right)}{\left(1+\left|\nabla u_{\varepsilon}\right|^{2}\right)^{5 / 2}} .
\end{aligned}
$$


Notice that

$$
\begin{aligned}
\Delta^{S} \frac{\left(u_{\varepsilon}\right)_{t}^{2}}{2} & =\Delta \frac{\left(u_{\varepsilon}\right)_{t}^{2}}{2}-\frac{\left(\nabla u_{\varepsilon}, \nabla^{2} \frac{\left(u_{\varepsilon}\right)_{t}^{2}}{2} \nabla u_{\varepsilon}\right)}{1+\left|\nabla u_{\varepsilon}\right|^{2}} \\
& =\left(u_{\varepsilon}\right)_{t} \Delta\left(u_{\varepsilon}\right)_{t}+\left|\left(\nabla u_{\varepsilon}\right)_{t}\right|^{2}-\frac{\left(u_{\varepsilon}\right)_{i}\left(u_{\varepsilon}\right)_{j}\left(u_{\varepsilon}\right)_{t}\left(u_{\varepsilon}\right)_{t i j}+\left(u_{\varepsilon}\right)_{i}\left(u_{\varepsilon}\right)_{j}\left(u_{\varepsilon}\right)_{t i}\left(u_{\varepsilon}\right)_{t j}}{1+\left|\nabla u_{\varepsilon}\right|^{2}}
\end{aligned}
$$

We then get

$$
\begin{aligned}
& \frac{d}{d t} \frac{\left(u_{\varepsilon}\right)_{t}^{2}}{2}=\frac{\left(\nabla\left(\frac{\left(u_{\varepsilon}\right)_{t}^{2}}{2}\right), \nabla u_{\varepsilon}\right)}{\sqrt{1+\left|\nabla u_{\varepsilon}\right|^{2}}} k_{\varepsilon}+\Delta^{S}\left(\frac{\left(u_{\varepsilon}\right)_{t}^{2}}{2}\right)-2 \frac{\left(\nabla\left(\frac{\left(u_{\varepsilon}\right)_{t}^{2}}{2}\right), \nabla\left(\frac{\left|\nabla u_{\varepsilon}\right|^{2}}{2}\right)\right)}{1+\left|\nabla u_{\varepsilon}\right|^{2}} \\
& +2 \frac{\left(\nabla\left(\frac{\left(u_{\varepsilon}\right)_{t}^{2}}{2}\right), \nabla u_{\varepsilon}\right)\left(\nabla\left(\frac{\left|\nabla u_{\varepsilon}\right|^{2}}{2}\right), \nabla u_{\varepsilon}\right)}{\left(1+\left|\nabla u_{\varepsilon}\right|^{2}\right)^{2}}+\frac{\left(\nabla u_{\varepsilon},\left(\nabla u_{\varepsilon}\right)_{t}\right)^{2}}{1+\left|\nabla u_{\varepsilon}\right|^{2}}-\left|\left(\nabla u_{\varepsilon}\right)_{t}\right|^{2}+\left(u_{\varepsilon}\right)_{t}^{2} \partial_{u} k_{\varepsilon} .
\end{aligned}
$$

Note that the last term is nonpositive by definition of $k_{\varepsilon}$.

In order to apply Lemma 1 , we have to show the inequality

$$
-\frac{\left(\nabla u_{\varepsilon},\left(\nabla u_{\varepsilon}\right)_{t}\right)^{2}}{1+\left|\nabla u_{\varepsilon}\right|^{2}}+\left|\left(\nabla u_{\varepsilon}\right)_{t}\right|^{2} \geqslant 0 .
$$

It is enough to note that, since the solution exists for all times and it is smooth, the term $\nabla\left(\frac{\left|\nabla u_{\varepsilon}\right|^{2}}{2}\right)$ is bounded on each $[0, T]$ (the bound depends on $T$ and $\varepsilon$ but is enough to apply the lemma). In addition, every factor containing $\nabla\left(\left(u_{\varepsilon}\right)_{t}^{2} / 2\right)$ also contains $\nabla u_{\varepsilon}$, hence the assumptions of Lemma 1 are satisfied for every $T>0$, and this concludes the proof.

From Propositions 9 and 10, we deduce the following result.

Proposition 11 If $u_{0}$ is $C$-Lipschitz in space for some $C>0$, and has bounded mean curvature, then the solution $u_{\varepsilon}$ of the approximate problem (28) is $C$-Lipschitz in space and Lipschitz in time with constant

Moreover, the following inequalities hold

$$
\left\|\sqrt{1+\left|\nabla u_{0}\right|^{2}} \operatorname{div}\left(\frac{\nabla u_{0}}{\sqrt{1+\left|\nabla u_{0}\right|^{2}}}\right)\right\|_{L^{\infty}\left(\mathbb{R}^{d}\right)} .
$$

$$
\psi_{\varepsilon}^{-}(x)-\varepsilon \leqslant u_{\varepsilon}(x, t) \leqslant \psi_{\varepsilon}^{+}(x)+\varepsilon .
$$

Proof. The Lipschitz bounds of the solution are clear (it is Proposition 9 and 10).

In order to prove the second assertion, let us notice that by (27) and the definition of $k_{\varepsilon}$, we have

$$
k_{\varepsilon}\left(x, \psi_{\varepsilon}^{-}-\varepsilon\right)=2 N \geqslant\left\|\sqrt{1+\left|\psi_{\varepsilon}^{-}\right|^{2}} \operatorname{div}\left(\frac{\psi_{\varepsilon}^{-}}{\sqrt{1+\left|\psi_{\varepsilon}^{-}\right|^{2}}}\right)\right\|_{L^{\infty}\left(\mathbb{R}^{d}\right)},
$$

so that $\psi_{\varepsilon}^{-}-\varepsilon$ is a subsolution of (28). By the parabolic comparison principle (as in Proposition 3), we deduce that

$$
\psi_{\varepsilon}^{-}-\varepsilon \leqslant u_{\varepsilon} .
$$

The same argument shows the other inequality in (33). 
Conclusion of the proof of Theorem 2. Since the solutions $u_{\varepsilon}$ are equi-Lipschitz in space and time, they converge uniformly, as $\varepsilon \rightarrow 0$, to a limit function $u$ which is also Lipschitz continuous on $\mathbb{R}^{d} \times[0,+\infty)$.

Equation (33) yields

$$
\psi^{-} \leqslant u \leqslant \psi^{+},
$$

and Proposition 8 gives that $u$ is a viscosity solution of (A1).

Concerning the regularity of $u$, we proved that $\left(u_{\varepsilon}\right)_{t}$ and $\nabla u_{\varepsilon}$ are bounded on $[0, T]$, for any $T$ in the approximate problem. This gives a bound on the mean curvature of the approximate solution. This bound does not depend on $\varepsilon$ and remains true for the viscosity solution. As a result, the exact solution has bounded mean curvature and bounded gradient, which shows that $\Delta u$ is $L^{\infty}$ and, by elliptic regularity theory, $u$ is also in $W^{2, p}$ for any $p>1$, and so $C^{1, \alpha}$ for every $\alpha<1$ (see [19] for details).

We can also directly apply to the solution $u$ a regularity result by Shahgholian (see [21, 23] and Theorem 4 below), which implies that $u$ is in fact of class $\mathrm{e}^{1,1}$. This concludes the proof of Theorem 2.

\section{Proof of Theorem 3}

We compute the evolution of the area of the graph of $u$ :

$$
\frac{d}{d t} \int_{Q} \sqrt{1+|\nabla u|^{2}}=\int_{Q} \frac{\left(\nabla u_{t}, \nabla u\right)}{\sqrt{1+|\nabla u|^{2}}}=-\int_{Q} u_{t} \operatorname{div}\left(\frac{\nabla u}{\sqrt{1+|\nabla u|^{2}}}\right) .
$$

Notice that, for almost every $t>0, u_{t}(t, x)=0$ almost everywhere on the contact set. Indeed, for almost every $t, u_{t}$ exists for almost every $x \in Q$. If $u(x, t)=\psi^{ \pm}(x)$, then $u-\psi^{ \pm}$reaches an extremum in $(x, t)$, which gives, $u_{t}(x, t)=0$. In particular, from (34) we get

$$
\frac{d}{d t} \int_{Q} \sqrt{1+|\nabla u|^{2}}=-\int_{Q} u_{t}\left(\frac{u_{t}}{\sqrt{1+|\nabla u|^{2}}}\right) .
$$

Integrating this equality in time, we obtain

$$
\left.\int_{Q} \sqrt{1+|\nabla u|^{2}}\right|_{0} ^{T}=\int_{0}^{T} \int_{Q}-\frac{u_{t}^{2}}{\sqrt{1+|\nabla u|^{2}}} .
$$

which shows that

$$
\int_{0}^{T} \int_{Q} u_{t}^{2}
$$

is uniformly bounded in $T$. As a result $u_{t} \in L^{2}\left(\mathbb{R}^{+}, Q\right)$ so $u$ is in $H^{1}\left(Q, B_{R}\right)$.

Since $\left\|u_{t}\right\|_{L^{2}(Q)}$ is $L^{2}\left(\mathbb{R}^{+}\right)$, there exists a sequence $t_{n} \rightarrow \infty$ such that

$$
\left\|u_{t}\right\|_{L^{2}(Q)}\left(t_{n}\right) \underset{n \rightarrow \infty}{\longrightarrow} 0
$$

In addition, $u\left(t_{n}\right)$ is equi Lipschitz and converges uniformly on compact sets to some $u_{\infty}$ which therefore satisfies in the viscosity sense

$$
\sqrt{1+|\nabla u|^{2}} \operatorname{div}\left(\frac{\nabla u}{\sqrt{1+\nabla u^{2}}}\right)=0
$$


with obstacles $\psi^{ \pm}$(see Appendix 5).

REMARK By [17], $u_{\min }$ is analytic out of the (closed) contact set $\left\{u_{\min }=\psi^{ \pm}\right\}$.

\section{Appendix A. Viscosity solutions with obstacles}

\section{A.1 Definition of viscosity solution}

Given an open subset $B$ of $\mathbb{R}^{d}$, let $u_{0}, \psi^{+}$and $\psi^{-}$be three Lipschitz functions $B \rightarrow \mathbb{R}$ such that

$$
\psi^{-}(x, 0) \leqslant u_{0}(x) \leqslant \psi^{+}(x, 0) .
$$

We are interested in the viscosity solutions of the equation

$$
u_{t}=\sqrt{1+|\nabla u|^{2}} \operatorname{div}\left(\frac{\nabla u}{\sqrt{1+|\nabla u|^{2}}}\right), \quad u(x, 0)=u_{0}(x)
$$

with the constraint

$$
\psi^{-}(x) \leqslant u(x, t) \leqslant \psi^{+}(x) .
$$

Definition 1 (see $[5,20]$ ) We say that a function $u: B \times[0, T) \rightarrow \mathbb{R}$ is a viscosity subsolution of (A1) if $u$ satisfies the following conditions:

- $u$ is upper semicontinuous;

- $u(x, 0) \leqslant u_{0}(x)$;

-

$$
\psi^{-}(x) \leqslant u(x, t) \leqslant \psi^{+}(x)
$$

- for any $\left(x_{0}, t_{0}\right) \in \mathbb{R}^{d} \times \mathbb{R}^{+}$and $\varphi \in C^{2}$ such that $u-\varphi$ has a maximum at $\left(x_{0}, t_{0}\right)$ and $u\left(x_{0}, t_{0}\right)>\psi^{-}\left(x_{0}\right)$,

$$
u_{t} \leqslant \sqrt{1+|\nabla u|^{2}} \operatorname{div}\left(\frac{\nabla u}{\sqrt{1+|\nabla u|^{2}}}\right)
$$

Similarly, $u$ is a viscosity supersolution of (A1) if:

- $u$ is lower semicontinuous;

- $u(x, 0) \geqslant u_{0}(x)$;

- (A3) holds;

- for any $\left(x_{0}, t_{0}\right) \in \mathbb{R}^{d} \times \mathbb{R}^{+}$and $\varphi \in C^{2}$ such that $u-\varphi$ has a minimum at $\left(x_{0}, t_{0}\right)$ and $u\left(x_{0}, t_{0}\right)<\psi^{+}\left(x_{0}\right)$

$$
u_{t} \geqslant \sqrt{1+|\nabla u|^{2}} \operatorname{div}\left(\frac{\nabla u}{\sqrt{1+|\nabla u|^{2}}}\right) .
$$

We say that $u$ is a viscosity solution of (A1) if it is both a super and a subsolution. 


\section{A.2 Comparison principle}

In order to prove uniqueness of continuous viscosity solutions of (A1), we shall prove a comparison principle between solutions following [13, Theorem 4] (see also [4]).

PROPOSITION 12 If $u$ is a viscosity subsolution of (A1) on [0,T), $v$ is a viscosity supersolution, if $\psi^{ \pm}$are Lipschitz in space and if $u(x, 0) \leqslant v(x, 0)$, then $u(x, t) \leqslant v(x, t)$ for all $(x, t) \in$ $\mathbb{R}^{n} \times[0, T)$.

Proof. We will check that the proof of [13, Theorem 2.1] can be extended to the obstacle case. Notice first that the assumptions $(A .1)-(A .3)$ of [13, Theorem 2.1] are satisfied also in our case. Indeed, $(A .1)$ comes directly from the Lipschitz bound on $\psi^{ \pm}$and the constraint $\psi^{-} \leqslant u, v \leqslant \psi^{+}$ whereas $(A .2)$ and $(A .3)$ result from the assumed time zero comparison.

Let us show that [13, Proposition 2.3] also holds. Indeed, up to Equation (2.9) nothing changes. To continue the proof, using the same notation of [13, Proposition 2.3], we have to check that if

$$
\sup _{V}(w-\Psi)>0
$$

then the supremum is reached in the complementary of the contact set $\left\{u=\psi^{-}\right\} \cup\left\{v=\psi^{+}\right\}$.

Indeed, notice that if $u(x, t)=\psi^{-}(x)$, then, for all $x, y, t, s$,

$$
u(x, t)-v(y, s)=\psi^{-}(x)-v(y, s) \leqslant \psi^{-}(y)+L(|x-y|)-v(y, s) \leqslant L(|x-y|)
$$

since $v \geqslant \psi^{-}$. Hence, if $u(x, t)=\psi^{-}(x)$, with $K^{\prime}>L$, we must have $w-\Psi \leqslant 0$, so the supremum of $w-\Psi$ is attained in the complementary of $\left\{u=\psi^{-}\right\}$. One can show similarly that the supremum is reached in the complementary of $\left\{v=\psi^{+}\right\}$. Hence Proposition 2.3 of [13] holds.

From Proposition 2.4 to Lemma 2.7 of [13], every result holds without changes.

Concerning the proof of Theorem 2.1 of [13], the first assumption is

$$
\alpha=\limsup _{\theta \rightarrow 0}\{w(t, x, y),|| x-y \mid \leqslant \theta\}>0 .
$$

Then, Proposition 2.4 gives constants $\delta_{0}$ and $\gamma_{0}$ such that for all $\delta \leqslant \delta_{0}, \gamma \leqslant \gamma_{0}$ and $\varepsilon>0$, there holds

$$
\Phi(\hat{x}, \hat{y}, \hat{t}):=\sup _{\mathbb{R}^{d} \times \mathbb{R}^{d} \times[0, T)} \Phi(x, y, t)>\frac{\alpha}{2}
$$

with

$$
\Phi(t, x, y)=u(x, t)-v(y, t)-\frac{|x-y|^{4}}{4 \varepsilon}-\delta\left(|x|^{2}+|y|^{2}\right)-\frac{\gamma}{T-t}
$$

To conclude the proof, we only have to show that the maximum of $\Phi$ is once again attained on the complementary of $\left\{u=\psi^{-}\right\} \cup\left\{v=\psi^{+}\right\}$. In the same way as for Proposition 2.3, if $u(x, t)=$ $\psi^{-}(x)$, we can write

$$
\begin{aligned}
\Phi(t, x, y) & =u(x, t)-v(y, t)-\frac{|x-y|^{4}}{4 \varepsilon}-\delta\left(|x|^{2}+|y|^{2}\right)-\frac{\gamma}{T-t} \\
& \leqslant \psi^{-}(y)+L|x-y|-v(y, t) \leqslant L|x-y| .
\end{aligned}
$$

Thanks to Proposition $2.5,|\hat{x}-\hat{y}| \underset{\varepsilon \rightarrow 0}{\longrightarrow} 0$. So, with $\varepsilon$ sufficiently small (one can reduce the quantity $\varepsilon_{0}$ given by Proposition 2.6), $\Phi$ has its maximum out of $\left\{u=\psi^{-}\right\}$(and similarly out of $\left\{v=\psi^{+}\right\}$), which enables the application of Lemma 2.7 and gives a contradiction as in [13]. 


\section{A.3 Existence}

In this subsection, we prove the following result:

PROPOSITION 13 There exists a continuous viscosity solution to (A1).

We follow [5] to build a solution by means of the Perron's method. Let us state an obvious but useful proposition and a key lemma for applying Perron's method.

PROPOSITION 14 Let $u$ be a subsolution of the mean curvature motion for graphs (without obstacles) which satisfies $u \leqslant u^{+}$. Then, $u_{o b}:=u \vee u^{-}$is a subsolution of (A1) with obstacles (the same happens for $v$ supersolution and $v_{o b}=v \wedge u^{+}$).

In the sequel, we shall denote by $u^{*}$ (resp. $u_{*}$ ) the upper (resp. lower) semicontinuous envelope of a function $u$.

LEMMA 6 Let $\mathcal{F}$ be a family of subsolutions of (A1). We define

$$
U(x, t)=\sup \{u(x, t) \mid u \in \mathcal{F}\} .
$$

Then, $U^{*}$ is a subsolution of (A1).

The proof of the proposition and the lemma can be found in [5], Lemma 4.2 (with obvious changes due to the parabolic situation and obstacles).

A.3.1 Construction of barriers.. In the sequel, to claim that the initial condition is taken by the viscosity solution, we need to build barriers around the solution $u$. More precisely, we want to build a subsolution $w^{-}$such that $\left(w^{-}\right)^{*}(x, 0)=u_{0}(x)$ and a supersolution $w^{+}$such that $\left(w^{+}\right)_{*}(x, 0)=$ $u_{0}(x)$. To show this claim, let us begin by a simple fact.

Let

$$
g_{\alpha, b}^{a}(x)=-\sum \alpha_{i} \frac{(x-a)_{i}^{2}}{\sqrt{1+(x-a)_{i}^{2}}}+b
$$

for some $(a, b) \in \mathbb{R}^{d} \times \mathbb{R}$ and $\alpha_{i} \geqslant 0$ such that $g(x) \leqslant u_{0}(x)$. Note in particular that

$$
g_{\alpha, b}^{a}(x) \geqslant-\sum \alpha_{i}(x-a)_{i}^{2}+b \quad \text { and } \quad H\left(g_{\alpha, b}^{a}\right) \geqslant\left. H\left(g_{\alpha, b}^{a}\right)\right|_{t=0}=-2 \sum \alpha_{i}
$$

Then, it is easy to show (using Proposition 14) that the function

$$
v(x, t)=\left(g_{\alpha, b}^{a}(x)+\left(2 \sum_{i=1}^{n} \alpha_{i}+3 M\right) t\right) \vee \psi^{-}
$$

is a subsolution of (A1). Indeed, the curvature of $g_{\alpha, b}^{a}$ is smaller than $2 \sum_{i} \alpha_{i}$ and its gradient is bounded by 2 (so $\sqrt{1+|\nabla g|^{2}} \leqslant 3$ ).

Thanks to Lemma 6, the function

$$
w^{-}(x, t)=\left(\sup _{\substack{\left(\alpha_{i}\right), c \\ g_{\alpha, b}^{c} \leqslant u_{0}}}\left(g_{\alpha, b}^{a}(x)-2 \sum_{i=1}^{n} \alpha_{i} t-3 M t\right) \vee \psi^{-}\right)^{*}
$$


is a subsolution of (A1) (with obstacles).

It remains to show that $\left(w^{-}\right)^{*}(x, 0)=u_{0}(x)$. To see this, notice that since $u_{0}$ is Lipschitz and $u_{0} \geqslant \psi^{-}, u_{0}(x)=w^{-}(x, 0)$, yielding $u_{0}(x) \leqslant\left(w^{-}\right)^{*}(x, 0)$. But for all $t \geqslant 0, v(x, t) \leqslant u_{0}(x)$ so $w^{-}(x, t) \leqslant u_{0}(x)$. By continuity of $u_{0},\left(w^{-}\right)^{*}(x, t) \leqslant u_{0}(x)$, which shows that $\left(w^{-}\right)^{*}(x, 0)=$ $u_{0}(x)$, and $w^{-}$is a low barrier for solutions of (A1).

We build $w^{+}$in the same way.

A.3.2 Perron's method.. We use the classical Perron's method to build a solution of (A1) on $[0, T)$ for every $t>0$. Let us define

$$
W(x, t)=\sup \{u(x): u \text { is a subsolution of (A1) on }[0, T)\} .
$$

Since $\psi^{-}$is a subsolution, this set in non empty and $W$ is well defined. Every subsolution is less that $\psi^{+}$, so is $W$.

Thanks to Lemma $6, W^{*}$ is a subsolution of (A1) regardless the initial conditions. Applying the comparison principle (Proposition 12) to every subsolution $u$ and $w^{+}$gives

$$
\forall x, t, W(x, t) \leqslant w^{+}(x, t) .
$$

Considering the upper-semi-continuous envelopes, we get

$$
\forall x, t, W^{*}(x, t) \leqslant\left(w^{+}\right)^{*}(x, t)
$$

which immediately yields to

$$
W^{*}(x, 0)=u_{0}(x)
$$

Then, $W^{*}$ is a subsolution of (A1), hence $W^{*}=W$ which shows the upper semi-continuity of $W$.

We want to prove that $W$ is actually a solution of (A1). In order to do this, let us prove the following

LEMMA 7 Let $u$ be a subsolution of (A1). If $u_{*}$ fails to be a supersolution (regardless initial conditions) at some point $(\hat{x}, \hat{t})$ then there exists a subsolution $u_{\kappa}$ (regardless initial conditions) satisfying $u_{\kappa} \geqslant u$ and $\sup u_{\kappa}-u>0$ and such that $u(x, t)=u_{\kappa}(x, t)$ for $|x-\hat{x}|,|t-\hat{t}| \leqslant \kappa$.

Proof. Let us assume that $u_{*}$ fails to be a supersolution at $(0,1)$. Then there exists $(a, p, X) \in$ $\mathcal{S}^{2,-} u_{*}(0,1)$ with

$$
a+F(p, X)+k(0) \sqrt{1+p^{2}}<0
$$

Let us then define

$$
u_{\delta, \gamma}(x, t)=u_{*}(0,1)+\delta+(p, x)+a(t-1)+\frac{1}{2}(X x, x)-\gamma\left(|x|^{2}+t-1\right) .
$$

Thanks to the continuity of $F$ and $k, u_{\delta, \gamma}$ is a classical subsolution on $B_{r}(0,1)$ of $u_{t}+$ $F\left(D u, D^{2} u\right)+k(x) \sqrt{1+|\nabla u|^{2}}=0$ for $\delta, \gamma, r$ sufficiently small. By assumption,

$$
u(x, t) \geqslant u_{*}(x, t) \geqslant u_{*}(0,1)+a(t-1)+(p, x)+\frac{1}{2}(X x, x,+) o\left(|x|^{2}+|t-1|\right) .
$$


With $\delta=\gamma \frac{r^{2}+r}{8}$, we get $u(x, t)>u_{\delta, \gamma}(x, t)$ for small $r$ and $|x|,|t-1| \in\left[\frac{r}{2}, r\right]$. Reducing again $r$, we can assume that $u_{\delta, \gamma}<\psi^{+}$on $B_{r}$. Thanks to Lemma 6 ,

$$
\tilde{u}(x, t)=\left\{\begin{array}{c}
\max \left(u(x, t), u_{\delta, \gamma}(x, t)\right) \text { if }|x, t-1|<r \\
u(x) \text { otherwise }
\end{array}\right.
$$

is a subsolution of (A1) (with no initial conditions).

Finally, this lemma combined with the definition of $W$ proves that $W$ is in fact a solution of (A1) (the initial conditions were already checked).

\section{A.4 Regularity}

Proposition 15 The unique solution $u$ of (A1) is Lipschitz in space, with the same constant as $u_{0}, \psi^{ \pm}$.

Proof. We will prove that $u_{z}(x, t)=u(x+z, t)-L|z|$ is in fact a subsolution of (A1). The Lipschitz bound is then straightforward (using the comparison principle).

To begin, we notice that $u(x+z, t)-L(|z|) \leqslant u^{+}(x, t)$ and $u(x+z, 0)-L|z| \leqslant u_{0}(x+z)-$ $L|z| \leqslant u_{0}(x)$.

Assume now that $\varphi$ is any smooth function which is greater than $u_{z}$ with equality at $(\hat{x}, \hat{t})$. Then, either, $u_{z}(\hat{x}, \hat{t})=\psi^{-}(\hat{x}, \hat{t})$ and nothing has to be done, or $u_{z}(\hat{x}, \hat{t})>\psi^{-}(\hat{x}, \hat{t})$. In the second alternative, one can write

$$
u(\hat{x}+t, \hat{t})>\psi^{-}(\hat{x})=\psi^{-}(\hat{x}+z)+\left(\psi^{-}(\hat{x})-\psi^{-}(\hat{x}+z),\right.
$$

so

$$
u(\hat{x}+z, \hat{t})>\psi^{-}(\hat{x}+z)+\underbrace{\psi^{-}(\hat{x})-\psi^{-}(\hat{x}+z)+L|z|}_{\geqslant 0} \geqslant u^{-}(\hat{x}+z, \hat{t}) .
$$

As $u$ is a subsolution at $(\hat{x}+z, \hat{t})$ and $u(x+z, t) \leqslant \varphi(x, t)+L|z|$ with equality at $(\hat{x}+z, \hat{t})$, one can write with $y=x+z, s=t$,

$$
u(y, t) \leqslant \varphi(y-z, s)+L|z|:=\phi(y, s),
$$

with equality at $(\hat{y}, \hat{s})$ which gives

$$
\phi_{t}+F\left(D \phi(\hat{x}, \hat{t}), D^{2} \phi(\hat{x}, \hat{t})\right) \leqslant 0
$$

Since the derivatives of $\phi$ and $\varphi$ are the same, we deduce

$$
\varphi_{t}+F\left(D \varphi, D^{2} \varphi\right) \leqslant 0
$$

what was expected.

REMARK With the same arguments, one can prove that

$$
\forall \delta>0, \quad \forall x, t, \quad|u(x, t+\delta)-u(x, t)| \leqslant \sup _{x}|u(x, \delta)-u(x, 0)| .
$$


We now present a general regularity result by Shahgholian [23] which applies to viscosity solutions for parabolic equations with obstacles.

TheOREM 4 ( [21], Th. 4.1) Let $Q^{+}:=\left\{(x, t) \in \mathbb{R}^{d} \times \mathbb{R}:|x|<1, t \in[0,1)\right\}$ and $H(u)=$ $F\left(D^{2} u, D u\right)-u_{t}$ where $F$ is uniformly elliptic. Let $u$ be a continuous viscosity solution of

$$
\begin{aligned}
(u-\psi) H(u) & =0, \\
H(u) & \leqslant 0, \\
u & \geqslant \psi,
\end{aligned}
$$

in $Q^{+}$, with boundary data

$$
u(x, t)=g(x, t) \geqslant \psi(x, t) \quad \text { on }\{|x|=1\} \cup\{t=0\} .
$$

Assume that $\psi \in C^{1,1}\left(Q^{+}\right)$and $g$ is continuous. Then, $u \in C^{1,1}$ on every compact subset of $Q^{+}$.

It has to be noticed $H=F-\partial_{t}$ where $F\left(D^{2} u, D u\right)=-\sqrt{1+|\nabla u|^{2}} \operatorname{div}\left(\frac{\nabla u}{\sqrt{1+|\nabla u|^{2}}}\right)$ satisfies all the assumptions of [23], 1.3. Indeed, the uniform ellipticity is provided by the Lipschitz bound obtained in previous subsection.

Moreover, the viscosity solution $u$ of (A1) satisfies (A7) and (A8) on every cylinder $Q_{r}^{+}\left(x_{0}\right):=$ $\left\{\left|x-x_{0}\right| \leqslant r, t \in\left[t_{0}, t_{0}+r\right)\right\}$ such that $r$ is chosen sufficiently small in order to have either $Q_{r}^{+}\left(x_{0}\right) \cap\left\{u=\psi^{+}\right\}=\emptyset$ or $Q_{r}^{+}\left(x_{0}\right) \cap\left\{u=\psi^{-}\right\}=\emptyset$. In the second alternative, change every sign in the equations.

Applying Theorem 4 we get a $C^{1,1}$ bound for $u$ on every compact subset of $Q_{r}^{+}\left(x_{0}\right)$. To show that $u$ is $C^{1,1}$ in the whole space, just cover $\mathbb{R}^{d} \times \mathbb{R}^{+}$with such $Q_{r}^{+}\left(x_{i}\right)$.

\section{REFERENCES}

1. Almeida, L., Chambolle, A. \& Novaga, M., Mean curvature flow with obstacles. Ann. Inst. H. Poincaré Anal. Non Linéaire 29 (2012), 667-681. Zb11252 . 49072 MR2971026

2. Almgren, F., TAYlor, J.-E. \& WAng, L., Curvature-driven flows: a variational approach. SIAM J. Control Optim. 31 (1993), 387-438. Zb10783. 35002 MR1205983

3. BRAKKe, K. A., The motion of a surface by its mean curvature, volume 20 of Mathematical Notes. Princeton University Press, Princeton, N.J., 1978. Zbl0386. 53047 MR0485012

4. Chen, Y. G., Giga, Y. \& Goto, S., Uniqueness and existence of viscosity solutions of generalized mean curvature flow equations. J. Differential Geom. 33 (1991), 749-786. Zb10696. 35087 MR1100211

5. Michael G. Crandall, Hitoshi Ishii, and Pierre-Louis Lions. User's guide to viscosity solutions of second order partial differential equations. Bull. Amer. Math. Soc. (N.S.), 27(1):1-67, 1992. Zb10755.35015 MR1118699

6. Cesaroni, A. \& Novaga, M., Long-time behavior of the mean curvature flow with periodic forcing. Comm. Partial Differential Equations 38 (2013), 780-801. Zbl1288. 35071 MR3046293

7. Cesaroni, A., Novaga, M. \& Valdinoci, E., Curve shortening flow in heterogeneous media. Interfaces Free Bound. 13 (2011), 485-505. Zb11261. 35079 MR2863468

8. Ecker, K. \& Huisken, G., Mean curvature evolution of entire graphs. Ann. of Math. 130 (1989), 453471. Zb10696. 53036 MR1025164

9. ECKER, K. \& HUISKEN, G., Interior estimates for hypersurfaces moving by mean curvature. Invent. Math. 105 (1991), 547-569. Zb10707.53008 MR1117150 
10. Ecker, K. \& Huisken, G., Parabolic methods for the construction of spacelike slices of prescribed mean curvature in cosmological spacetimes. Comm. Math. Phys. 135 (1991), 595-613. Zb10721.53055 MR1091580

11. Evans, L. C. \& Spruck, J., Motion of level sets by mean curvature. I. J. Differential Geom. 33 (1991), 635-681. Zb10726.53029 MR1100206

12. Elliott, C. M., Stinner, B. \& Venkataraman, C., Modelling cell motility and chemotaxis with evolving surface finite elements. Journal of The Royal Society Interface (2012), page rsif20120276.

13. Giga, Y., Goto, S., IshiI, H. \& SAto, M.-H., Comparison principle and convexity preserving properties for singular degenerate parabolic equations on unbounded domains. Indiana Univ. Math. J. 40 (1991), 443-470. Zb10836. 35009 MR1119185

14. Gage, M. \& Hamilton, R. S., The heat equation shrinking convex plane curves. J. Differential Geom. 23 (1986), 69-96. Zb10621.53001 MR0840401

15. Huisken, G., Flow by mean curvature of convex surfaces into spheres. J. Differential Geom. 20 (1984), 237-266. Zb10556.53001 MR0772132

16. Huisken, G., Asymptotic behavior for singularities of the mean curvature flow. J. Differential Geom. 31 (1990), 285-299. Zb10694.53005 MR1030675

17. Ilmanen, T., Sternberg, P. \& Ziemer, W. P., Equilibrium solutions to generalized motion by mean curvature. J. Geom. Anal. 8 (1998), 845-858. Dedicated to the memory of Fred Almgren. Zb10941. 35028 MR1731065

18. Luckhaus, S. \& Sturzenhecker, T., Implicit time discretization for the mean curvature flow equation. Calc. Var. Partial Differential Equations 3 (1995), 253-271. Zbl0821. 35003 MR1386964

19. LunARDI, A., Analytic semigroups and optimal regularity in parabolic problems. Modern Birkhäuser Classics. Birkhäuser/Springer Basel AG, Basel, 1995. [2013 reprint of the 1995 original] Zbl1261. 35001 MR3012216

20. Mercier, G., Mean curvature flow with obstacles: A viscosity approach. Preprint, 2014.

21. Petrosyan, A. \& Shahgholian, H., Parabolic obstacle problems applied to finance. In Recent developments in nonlinear partial differential equations, volume 439 of Contemp. Math., pages 117-133. Amer. Math. Soc., Providence, RI, 2007. Zb11137.35082 MR2359024

22. Protter, M. H. \& Weinberger, H. F., Maximum principles in differential equations. Springer-Verlag, New York, 1984. Corrected reprint of the 1967 original. Zbl0549.35002 MR0762825

23. Shahgholian, H., Free boundary regularity close to initial state for parabolic obstacle problem. Trans. Amer. Math. Soc. 360 (2008), 2077-2087. Zbl1221. 35454 MR2366975

24. Spadaro, E., Mean-convex sets and minimal barriers. Preprint, 2011. 